\title{
Diffusion à longue distance
}

\author{
H. Jobic
}

Institut de Recherches sur la Catalyse, CNRS, 2 avenue Albert Einstein, 69626 Villeurbanne, France

\begin{abstract}
Résumé. La diffusion quasi-élastique des neutrons permet d'étudier le mouvement à longue distance d'atomes ou de molécules en phase condensée ou adsorbée. L'un des avantages de la méthode est de pouvoir mesurer directement le coefficient de diffusion dans un domaine de distance où la loi de Fick est respectée. D'autre part, en utilisant un modèle de diffusion par sauts, on peut déterminer les distances et les temps de sauts élémentaires. Dans le cas d'une diffusion isotrope, les différents modèles de saut existants : Chudley - Elliott, Singwi - Sjölander et Hall - Ross sont passés en revue, et un nouveau modèle impliquant une délocalisation de la particule sur son site est présenté. Les cas de diffusion unidimensionnelle normale et à la file indienne sont examinés. Les exemples d'application concernent des molécules adsorbées dans des zéolithes : hydrogène dans les zéolithes NaA et NaX, alcanes dans la structure MFI, et systèmes unidimensionnels.
\end{abstract}

\section{INTRODUCTION}

Les problèmes de diffusion interviennent dans tous les états de la matière : gaz, liquide, et solide, et plus spécifiquement dans des domaines aussi variés que les polymères, les interfaces, la catalyse, etc. Les échelles de temps mises en jeu varient de façon considérable. Pour caractériser ce phénomène, de multiples techniques ont été utilisées ou mises au point spécialement. Parmi ces techniques, la diffusion quasi-élastique des neutrons (DQEN) a surtout été appliquée à l'étude de la diffusion (i) de l'hydrogène dans les métaux, (ii) de molécules adsorbées dans des zéolithes, et (iii) d'ions dans des oxydes ou dans des électrolytes. Dans un texte analogue en Anglais, on éviterai la confusion entre diffusion des neutrons (scattering) et mobilité des particules (diffusion)!

\section{LOIS DE FICK}

La théorie du mouvement Brownien a été étudiée de façon indépendante et différente par Einstein, Smoluchowski, et Langevin. Nous nous contenterons ici de la version simplifiée de la théorie d'Einstein. Le mouvement Brownien, purement aléatoire, est directement relié aux équations phénoménologiques de Fick, dont les travaux remontent en 1855 [1]. La première loi de Fick énonce simplement que le flux est proportionnel au gradient de concentration, le long d'une direction donnée :

$$
J=-D \frac{d c}{d x}
$$

Le flux de matière $\mathrm{J}$ a ainsi tendance a diminuer le gradient de concentration. En raisonnant dans les trois directions de l'espace, on peut écrire

$$
\mathbf{J}=-D \operatorname{grad} c
$$

Cette première loi de Fick définit le coefficient de diffusion, $\mathrm{D}$, à un niveau macroscopique. La dimension de $\mathrm{D}$ correspond à une surface divisée par le temps (il est le plus souvent donné en $\mathrm{m}^{2} \mathrm{~s}^{-1}$ ou en $\mathrm{cm}^{2} \mathrm{~s}^{-1}$ ). Dans un système isotrope, par exemple un liquide ou un réseau cubique, le coefficient de diffusion a la même valeur dans toutes les directions. Dans les systèmes anisotropes, le coefficient de diffusion est un tenseur symétrique, de rang 2.

La première loi de Fick est bien adaptée pour les méthodes stationnaires, comme les mesures de perméation. Cependant si la concentration à un endroit donné dépend du temps, il est plus aisé d'utiliser la seconde loi de Fick, qui dérive d'une équation de continuité (conservation de matière) : 


$$
\frac{\partial c}{\partial t}=-\nabla \mathbf{J}
$$

En utilisant les équations (2) et (3), la seconde loi de Fick s'écrit

$$
\frac{\partial c}{\partial t}=\operatorname{div}(D \operatorname{grad} c)
$$

Dans le cas où $\mathrm{D}$ est une constante, on obtient

$$
\frac{\partial c}{\partial t}=D \nabla^{2} c
$$

On souhaite alors déterminer la concentration en fonction de la position et du temps. Selon les conditions de l'expérience, la géométrie de l'échantillon, etc., les conditions initiales et les conditions aux limites sont différentes, de telle sorte que les solutions de cette équation différentielie sont très variées [2].

Dans de nombreuses techniques macroscopiques, on établit effectivement un gradient de concentration et on mesure alors un transfert de matière que l'on caractérise par un coefficient de transport. On peut aussi faire des mesures en suivant le déplacement d'espèces marquées, il s'agit alors d'autodiffusion ; le marquage pouvant être réalisé en utilisant des isotopes radioactifs. On mesure également l'autodiffusion par DQEN ou par RMN en gradient de champ pulsé. On se place alors dans des conditions d'équilibre et on suit le déplacement d'atomes ou de molécules en fonction du temps.

Dans ce chapitre consacré à la diffusion à longue distance étudiée par DQEN, nous nous limiterons à l'autodiffusion que l'on peut mesurer par diffusion incohérente des neutrons. La diffusion cohérente des neutrons permet d'accéder au coefficient de transport d'atomes ou de molécules, mais les expériences sont beaucoup moins nombreuses, car il faut alors séparer les contributions cohérentes et incohérentes [34].

\section{DIFFUSION ISOTROPE MESUREE PAR DQEN}

\subsection{Diffusion continue}

En DQEN, on se situe à l'échelle microscopique et on a coutume d'utiliser la fonction de corrélation de van Hove, $G(r, t)$. Comme nous ne traiterons que de la diffusion d'espèces hydrogénées, l'intensité diffusée par l'échantillon sera essentiellement incohérente [5]. On suivra ainsi la trajectoire d'une seule particule, qui est décrite par la fonction d'autocorrélation $G_{S}(r, t)$. Pour faire le lien avec la loi de Fick, il faut considérer que la fonction d'autocorrélation peut s'exprimer en fonction d'une densité de probabilité spatiale. L'équation (5) s'écrit alors :

$$
\frac{\partial G_{S}(\mathbf{r}, t)}{\partial t}=D \nabla^{2} G_{S}(\mathbf{r}, t)
$$

D désignera dorénavant le coefficient d'autodiffusion. Dans un liquide, il suffit de regarder la diffusion à une échelle de quelques dizaines de diamètres moléculaires pour que l'approximation Gaussienne soit correcte pour $G_{S}(\mathbf{r}, \mathfrak{t})$. Le milieu peut alors être considéré comme homogène et continu. En considérant une Gaussienne normalisée ayant l'expression suivante

$$
G_{S}(\mathbf{r}, t)=\frac{1}{(4 \pi D t)^{3 / 2}} \exp \left(-\frac{r^{2}}{4 D t}\right)
$$

on a bien une solution pour l'équation (6), qui remplit la condition initiale $G_{S}(r, 0)=\delta(\mathbf{r})$. De plus, on obtient comme valeur pour le déplacement carré moyen

$$
<\mathbf{r}^{2}(t)>=\int r^{2} G_{S}(\mathbf{r}, t) d \mathbf{r}=\frac{4 \pi}{(4 \pi D t)^{3 / 2}} \int_{0}^{\infty} r^{4} \exp \left(-\frac{r^{2}}{4 D t}\right) d r=6 D t
$$

Ainsi, quand le mouvement de diffusion suit la loi de Fick, le déplacement carré moyen est proportionnel au temps (nous verrons que dans le cas particulier de la diffusion à la file indienne, le déplacement carré moyen est proportionnel à la racine carrée du temps). Le parallèle avec le mouvement Brownien, ou aléatoire, est immédiat. L'autodiffusion d'un atome d'hydrogène dans un réseau cristallin 
ou d'une molécule dans une zéolithe correspond au problème de la marche au hasard. Par exemple, on considère une particule qui se déplace par sauts successifs dans un réseau cubique. La distance de saut $\ell$ est constante ainsi que la fréquence de saut $(1 / \tau)$ où $\tau$ est le temps entre les sauts. Dans ces conditions, Einstein a établi la relation [6]

$$
D=\frac{\ell^{2}}{6 \tau}
$$

Cette expression permet de relier des sauts à l'échelle atomique avec un coefficient de diffusion macroscopique. Elle est analogue à la relation obtenue à partir de la loi de Fick, équation (8). II apparaît ainsi que pour un mouvement aléatoire, une particule mettra 100 fois plus de temps pour parcourir une distance 10 fois plus grande.

Parmi toutes les méthodes disponibles pour mesurer les mécanismes de diffusion, l'avantage de la DQEN, de part sa gamme en Q, est double : on peut à la fois (i) déterminer les grandeurs $\ell$ et $\tau$ des sauts élémentaires, donc en déduire le coefficient de diffusion d'Einstein, et (ii) mesurer directement $\mathrm{D}$ dans un domaine de distance où la loi de Fick est respectée.

En DQEN, l'intensité diffusée par les espèces hydrogénées est proportionnelle à la fonction de diffusion incohérente, $S_{\text {inc }}(\mathbf{Q}, \omega)$, qui est la transformée de Fourier dans l'espace et dans le temps de $\mathrm{G}_{\mathrm{S}}(\mathbf{r}, \mathrm{t})[5]$.

Une première transformée de Fourier spatiale conduit à la fonction de diffusion intermédiaire

$$
I_{S}(\mathbf{Q}, t)=\int \exp (i \mathbf{Q} \cdot \mathbf{r}) G_{S}(\mathbf{r}, t) d \mathbf{r}=\exp \left(-D Q^{2} t\right)
$$

et le spectre en énergie est la transformée de Fourier temporelle de $I_{s}(\mathbf{Q}, t)$

$$
S_{i n c}(\mathbf{Q}, \omega)=\int \exp (-i \omega t) I_{S}(\mathbf{Q}, t) d t=\frac{1}{\pi} \frac{D Q^{2}}{\omega^{2}+\left(D Q^{2}\right)^{2}}
$$

Le profil du spectre est une Lorentzienne, centrée à $\omega=0$, de demi-largeur à mi-hauteur (HWHM) $\mathrm{DQ}^{2}$. En déterminant la largeur des spectres obtenus pour différentes valeurs de $Q$, on doit obtenir une droite en fonction de $Q^{2}$. Cependant, ce comportement n'est observé qu'aux petites valeurs de $Q$, correspondant aux grandes distances (Figure 1). Aux plus grandes valeurs de $Q$, un écart à cette droite est généralement observé, car le milieu n'est plus continu. Cet écart est caractéristique d'une diffusion par sauts.

Un autre argument a été invoqué pour expliquer le rétrécissement du pic par rapport à la loi en $\mathrm{DQ}^{2}$ [7]. Il souligne que la vitesse d'une molécule telle que l'on peut la déduire de l'équation (9)

$$
(6 \mathrm{D} / \mathrm{t})^{1 / 2}
$$

ne peut être supérieure à la vitesse dans le gaz parfait :

$$
v=\left(\frac{3 k T}{m}\right)^{1 / 2}
$$

En fait, ce raisonnement ne s'applique pas aux cas que l'on traitera ici. Par exemple, le coefficient de diffusion d'une molécule de méthane dans une zéolithe à canaux est de $10^{-8} \mathrm{~m}^{2} \mathrm{~s}^{-1}$, à $300 \mathrm{~K}$, la distance de saut élémentaire étant de $10 \AA$. Le temps entre les sauts est de 16,6 ps et la vitesse moyenne de $60 \mathrm{~m} / \mathrm{s}$. A partir de la théorie cinétique des gaz parfaits, on obtient une vitesse de $685 \mathrm{~m} / \mathrm{s}$ pour une molécule de méthane à $300 \mathrm{~K}$. Cette phase balistique, pour laquelle le déplacement carré moyen de la molécule est proportionnel à $t^{2}$, n'intervient donc pas dans les conditions expérimentales courantes. Le temps de transition entre les deux régimes : la phase balistique, et la diffusion normale au cours de laquelle le déplacement carré moyen est proportionnel au temps, équation (8), est de l'ordre de $10^{-13} \mathrm{~s}$, ce qui est en dehors du domaine quasi-élastique habituel. Cette phase balistique peut être mise en évidence aux temps courts par dynamique moléculaire, mais à l'échelle de temps de la DQEN, la molécule aura interagi avec la paroi ou avec les autres molécules.

\subsection{Diffusion par sauts}

Dans les cas qui nous concernent, l'écart à la loi de Fick est ainsi dû au caractère discontinu de la diffusion. Pour l'interprétation des résultats, on utilise des modèles de diffusion par sauts qui s'appliquent 

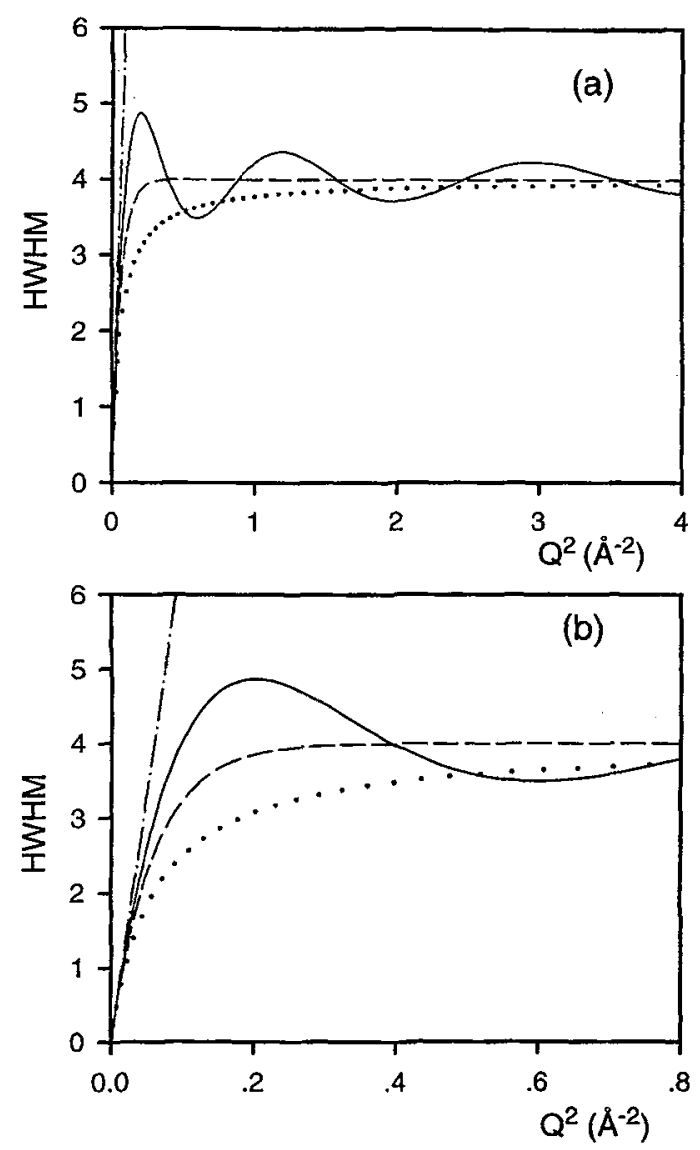

Figure 1. Elargissements calculés en fonction de $Q^{2}$ pour différents modèles : Chudley - Elliott (ligne continue), Hall - Ross (tirets), Singwi - Sjölander (pointillés) et Fick (tirets - points). Les calculs sont faits pour des mêmes valeurs de $\left\langle r^{2}\right\rangle=100 \AA^{2}$ et de $\tau$, donc pour le même coefficient de diffusion $D$.

à différents systèmes : hydrogène dans les métaux, liquides moléculaires, molécules adsorbées dans les zéolithes, etc. Il faut alors décrire les distances et les temps de sauts élémentaires.

3.2.1. Modèles existants : Chudley - Elliott, Singwi - Sjölander, Hall - Ross Un des premiers modèles de diffusion par sauts est celui de Chudley et Elliott [8]. Ce modèle a été initialement écrit pour des liquides où il subsiste un ordre à courte distance. En fait, il a trouvé de nombreuses applications dans différents domaines. Ce modèle est basé sur les hypothèses suivantes : un atome vibre sur un site pendant un temps $\tau$. Au bout de ce temps $\tau$, l'atome saute sur un autre site situé à une distance d (le temps de saut est beaucoup plus court que le temps de résidence), un atome ayant $\mathbf{n}$ possibilités de saut. Chudley et Elliott ont montré que dans ces conditions, la fonction de diffusion incohérente était aussi une Lorentzienne

$$
S(\mathbf{Q}, \omega)=\frac{1}{\pi} \frac{\Delta \omega(\mathbf{Q})}{\omega^{2}+(\Delta \omega(\mathbf{Q}))^{2}}
$$


la demi-largeur à mi-hauteur n'étant plus simplement $D Q^{2}$ mais de la forme

$$
\Delta \omega(\mathbf{Q})=\frac{1}{n \tau} \sum_{\mathbf{d}}[1-\exp (i \mathbf{Q} . \mathbf{d})]
$$

II faut noter que tous les sites sont supposés équivalents, si ce n'est pas le cas l'expression qui donne la largeur est plus compliquée [9]. Ce modèle s'applique bien à l'étude de la diffusion de l'hydrogène atomique adsorbé dans les métaux : les hydrures. Si l'on dispose d'un monocristal, on peut orienter le vecteur $\mathbf{Q}$ selon certaines directions cristallographiques. Dans le cas de poudres, l'équation (14) doit être moyennée numériquement pour tenir compte de toutes les orientations possibles des cristallites par rapport à $\mathbf{Q}$. Comme un vecteur se définit dans l'espace par 2 angles, une méthode triviale consiste à faire varier l'orientation de $\mathbf{Q}$ en incrémentant chaque angle. Une autre méthode plus rapide et plus précise revient à générer un plus petit nombre de points sur la sphère d'intégration [10,11]. C'est cette dernière méthode qui est utilisée en diffusion inélastique des neutrons, 52 ou 55 points se révélant suffisants. On obtient alors, pour chaque valeur de $\mathrm{Q}$, une somme de Lorentziennes de largeurs différentes. Aux petites valeurs de $\mathrm{Q}$, le profil obtenu est assimilable à une Lorentzienne, mais il s'en écarte pour les grandes valeurs de $\mathrm{Q}$, ainsi qu'il est montré sur la Figure 2. Cet exemple correspond à des sauts entre sites octaédriques d'un métal de type cfc (une distance de saut arbitraire de $3 \AA$ a été choisie). Le calcul de la largeur à mi-hauteur, représenté sur la Figure 3 en ligne continue, n'est théoriquement justifié que pour des valeurs de $Q$ allant jusqu'à $1 \AA^{-1}$ dans notre exemple, cependant il reste utile dans les cas où le profil ne diverge pas trop d'une Lorentzienne.
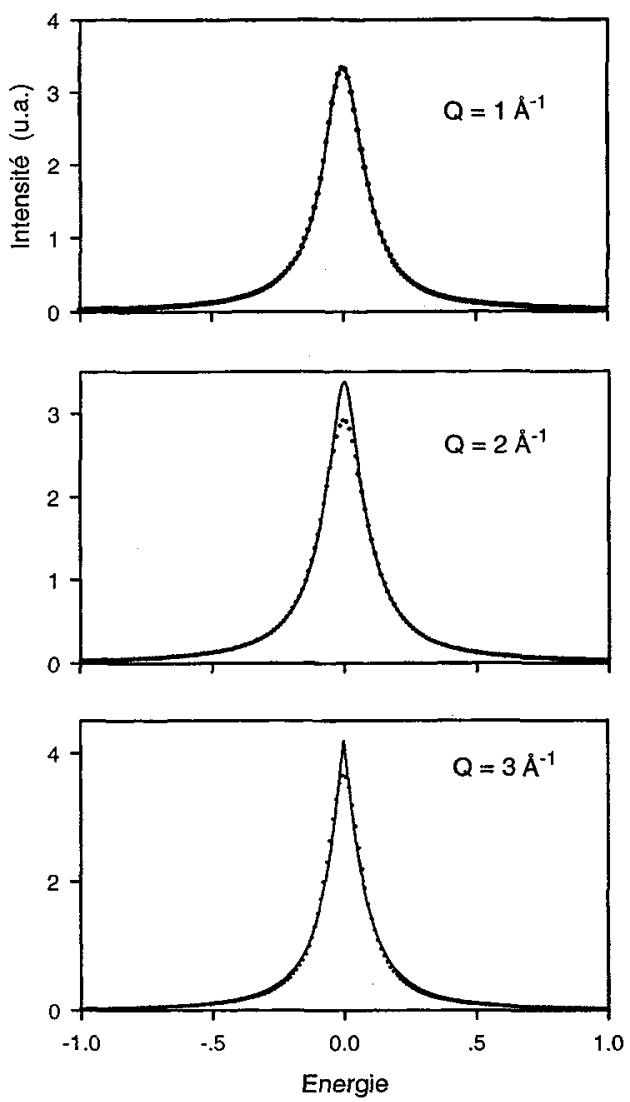

Figure 2. Spectres DQEN simulés, sans convolution avec la résolution instrumentale, pour des sauts entre sites octaédriques d'un réseau de Bravais (la distance de saut est de $3 \AA$ ). La ligne continue est obtenue après la moyenne de poudre, la ligne en pointillés correspond à une Lorentzienne ajustée sur le profil moyenné. 


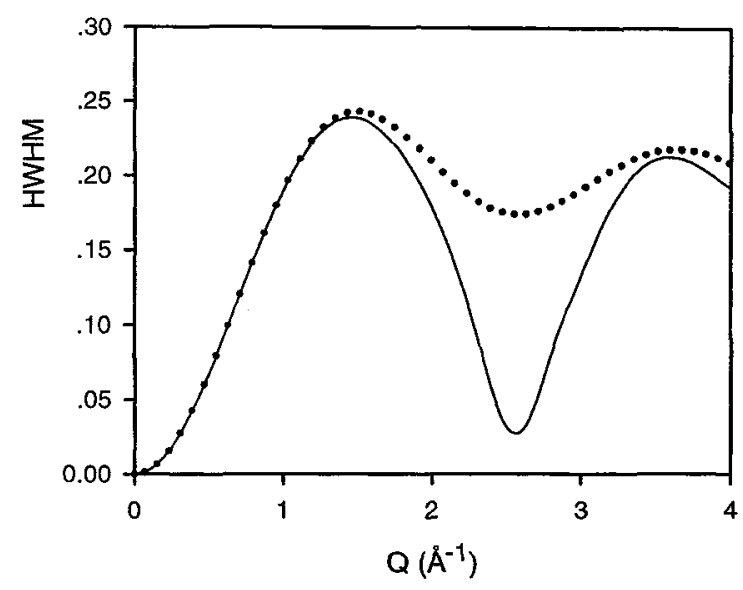

Figure 3. Calcul avec le modèle de Chudley - Elliott de l'élargissement en fonction de $Q$, pour des sauts entre sites octaédriques d'un réseau de Bravais (tous les sites sont équivalents, la distance de saut est de 3 Å). La ligne continue a été obtenue après une moyenne de poudre. La courbe en pointillés correspond au cas isotrope.

Chudley et Elliott ont également montré qu'en faisant l'hypothèse de sauts dans n'importe quelle direction, typiquement le cas d'un liquide, on avait alors la moyenne suivante :

$$
\begin{aligned}
\Delta \omega(Q) & =\frac{1}{\tau}\left(1-\frac{1}{2} \int_{0}^{\pi} d \theta \sin \theta \exp (i Q d \cos \theta)\right) \\
& =\frac{1}{\tau}\left(1-\frac{\sin (Q d)}{Q d}\right)
\end{aligned}
$$

Cette fonction est tracée sur la Figure 3 en ligne pointillée, pour une distance de saut de $3 \AA$. On peut noter qu'en moyennant seulement la largeur de la Lorentzienne, équation (15), on obtient un résultat proche de la ligne pointillée, ce qui est manifestement différent du résultat correct obtenu en moyennant les Lorentziennes, équation (14), surtout pour les grandes valeurs de $\mathrm{Q}$. au carré :

Pour un mouvement aléatoire, le déplacement carré moyen est proportionnel à la distance de saut

$$
<\mathbf{r}^{2}(t)>=N d^{2}
$$

$\mathrm{N}$ étant le nombre de sauts. Expérimentalement, on peut mesurer des élargissements pour des valeurs de $\mathrm{Q}$ proches de $0,1 \AA^{-1}$, ce qui correspond dans l'espace réel à des distances de $2 \pi / \mathrm{Q}$ de l'ordre de $60 \AA$. Pour des sauts de $3 \AA$, cela signifie que l'on sera capable de suivre le déplacement d'une particule après 400 sauts. En regardant à cette échelle, le vecteur d peut prendre n'importe quelle direction, l'approximation isotrope est justifiée et le produit $|\mathbf{Q} . \mathbf{d}|^{2}$ peut être remplacé par $\mathrm{Q}^{2} \mathrm{~d}^{2} / 3$. Les équations (15) et (16) ont alors la même limite pour les petites valeurs de $Q$ :

$$
\Delta \omega(Q)=\frac{Q^{2} d^{2}}{6 \tau}=D Q^{2}
$$

En utilisant la relation d'Einstein, équation (9), on retrouve ainsi la loi de Fick propre à un système macroscopique.

Un autre résultat remarquable obtenu par Chudley et Elliott (CE) est d'avoir montré que si la distance de saut n'était pas une constante, comme dans l'équation (16), mais présentait une distribution $\rho(r)$, on avait alors pour un système isotrope : 


$$
\Delta \omega(Q)=\frac{1}{\tau} \int\left(1-\frac{\sin (Q r)}{Q r}\right) \rho(r) d r / \int \rho(r) d r
$$

Comme nous n'utiliserons que des distributions normalisées, l'équation précédente peut s'écrire sous une forme simplifiée

$$
\Delta \omega(Q)=\frac{1}{\tau}\left[1-\int \frac{\sin (Q r)}{Q r} \rho(r) d r\right]
$$

Pour le modèle $C E$ habituel, cette distribution est de la forme $\delta(r-d)$. Les autres modèles qui ont été proposés dans la littérature peuvent tous être retrouvés à partir de l'équation (20). Ainsi, Egelstaff semble avoir été le premier à montrer que si l'on définissait une distribution de la forme $\rho(r)=r \exp (-$ $\mathrm{r} / \mathrm{r}_{0}$ ), on aboutissait à la version simplifiée du modèle de Singwi et Sjölander [12], en négligeant le temps de saut devant le temps de résidence [13]. Pour comparer les différents modèles, on utilisera plutôt pour le modèle SS une distribution normalisée représentée sur la Figure 4 en ligne pointillée

$$
\rho^{S S}(r)=\frac{r}{r_{0}^{2}} \exp \left(-\frac{r}{r_{0}}\right)
$$

La distance de saut carrée moyenne correspondant à cette distribution a pour valeur

$$
<r^{2}>=\int_{0}^{\infty} r^{2} \rho(r) d r=\frac{1}{r_{0}^{2}} \int_{0}^{\infty} r^{3} \exp \left(-\frac{r}{r_{0}}\right) d r=6 r_{0}^{2}
$$

A partir de l'équation (20), on obtient pour la largeur de la Lorentzienne

$$
\begin{aligned}
\Delta \omega(Q) & =\frac{1}{\tau}\left[1-\frac{1}{Q r_{0}^{2}} \int_{0}^{\infty} \sin (Q r) \exp \left(-\frac{r}{r_{0}}\right) d r\right] \\
& =\frac{1}{\tau}\left(1-\frac{1}{1+Q^{2} r_{0}^{2}}\right)=\frac{1}{\tau} \frac{Q^{2} r_{0}^{2}}{1+Q^{2} r_{0}^{2}}
\end{aligned}
$$

En utilisant l'équation (22), cette expression peut s'écrire

$$
\Delta \omega(Q)=\frac{1}{6 \tau} \frac{Q^{2}<r^{2}>}{1+Q^{2}<r^{2}>/ 6}
$$

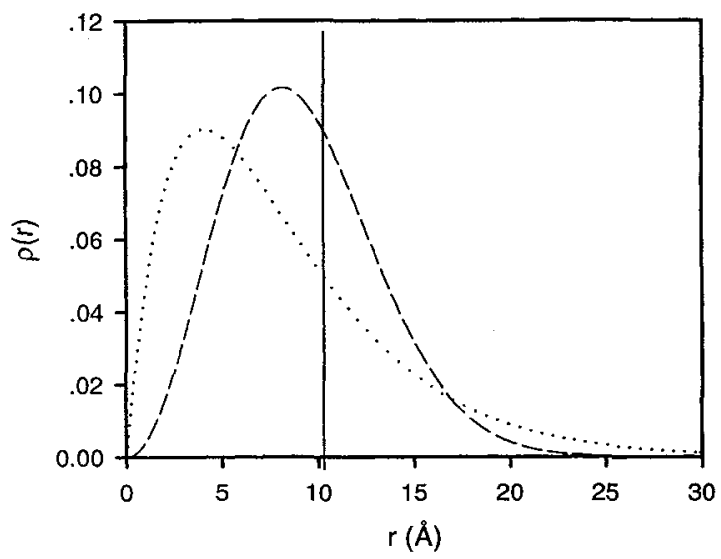

Figure 4. Distributions de sauts correspondant aux modèles de Chudley - Elliott (fonction $\delta$ à $10 \AA$ ), Singwi - Sjölander (ligne pointillée), et Hall - Ross (tirets). Les deux courbes sont calculées pour une même valeur du déplacement carré moyen : $\left\langle\mathrm{r}^{2}\right\rangle=$ $100 \AA^{2} ;$ on $a r_{0}=4,082 \AA$ pour $S S$ et $r_{0}=5,773 \AA$ pour $H R$. 
Quand $Q$ tend vers zéro, la largeur tend vers $\left\langle r^{2}>Q^{2} / 6 \tau\right.$, soit $D^{2}$, en utilisant une forme voisine de l'équation d'Einstein

$$
D=\frac{<r^{2}>}{6 \tau}
$$

la distance de saut carrée moyenne remplaçant la distance de saut au carré (cf. équation 9).

Le troisième modèle, qui a été publié par Hall et Ross (HR), est basé sur une distribution de la forme [7]

$$
\rho^{H R}(r)=\frac{2 r^{2}}{r_{0}^{3}(2 \pi)^{1 / 2}} \exp \left(-\frac{r^{2}}{2 r_{0}^{2}}\right)
$$

Cette distribution, qui est normalisée, est tracée sur la Figure 4 en ligne continue. Par rapport à la distribution de SS, le maximum se situe à une valeur de r plus grande, ce qui fait que la largeur (Figure 1) atteint sa valeur asymptotique plus rapidement.

La distance de saut carrée moyenne correspondant à la distribution de HR est :

$$
<r^{2}>=\int_{0}^{\infty} r^{2} \rho(r) d r=\frac{2}{r_{0}^{3}(2 \pi)^{1 / 2}} \int_{0}^{\infty} r^{4} \exp \left(-\frac{r}{r_{0}}\right) d r=3 r_{0}^{2}
$$

Pour ce modèle, on obtient comme élargissement :

$$
\begin{aligned}
\Delta \omega(Q) & =\frac{1}{\tau}\left[1-\frac{2}{Q r_{0}^{3}(2 \pi)^{1 / 2}} \int_{0}^{\infty} r \sin (Q r) \exp \left(-\frac{r^{2}}{2 r_{0}^{2}}\right) d r\right] \\
& =\frac{1}{\tau}\left[1-\exp \left(-\frac{Q^{2} r_{0}^{2}}{2}\right)\right]
\end{aligned}
$$

Il est intéressant de remarquer que Hall et Ross aboutissent à ce résultat par une démonstration différente [7]. En fait, comme les hypothèses de départ sont les mêmes pour tous ces modèles : processus markovien avec un seul temps de résidence, on peut toujours partir de l'équation (20), seule la distribution des distances de saut est différente.

Les largeurs des différents modèles de saut, représentées sur la Figure 1, correspondent aux distributions tracées sur la Figure 4. Ces largeurs ont toutes été calculées pour les mêmes valeurs de $\left\langle\mathrm{r}^{2}\right\rangle$ et de $\tau$, donc pour un même coefficient de diffusion $\mathrm{D}$. Cela se vérifie par le fait que tous ces modèles ont un domaine linéaire commun aux petites valeurs de $\mathrm{Q}$ : le domaine où la loi de Fick est vérifiée. On peut noter que seul le modèle de Chudley - Elliott a un comportement oscillatoire (Figure 1a), toutes les largeurs tendant vers $1 / \tau$ à grand $Q$.

3.2.2. Nouveau Modèle Dans certaines zéolithes, le site d'adsorption d'une molécule n'est pas toujours un point bien localisé mais il constitue parfois une région de l'espace délimitée par un canal ou une cavité de dimension moléculaire (exemple: Figure 5). Si l'on désigne par $d_{0}$ la distance entre deux sites et en reliant à $\mathrm{r}_{0}$ l'étendue de la délocalisation de la molécule sur un site (physiquement on doit avoir $\mathrm{r}_{0}<\mathrm{d}_{0}$ ), on peut alors proposer une nouvelle distribution de distances de sauts, toujours pour un système isotrope :

$$
\rho(r)=\frac{r}{d_{0} r_{0}(2 \pi)^{1 / 2}} \exp \left(-\frac{\left(r-d_{0}\right)^{2}}{2 r_{0}^{2}}\right)
$$

Cette distribution est normalisée, elle est représentée sur la Figure 6(a) pour différentes valeurs de $r_{0}$ et de $d_{0}$. La distance de saut carrée moyenne correspondant à ce modèle est donnée par

$$
<r^{2}>=\int_{0}^{\infty} r^{2} \rho(r) d r=\frac{1}{d_{0} r_{0}(2 \pi)^{1 / 2}} \int_{0}^{\infty} r^{3} \exp \left(-\frac{\left(r-d_{0}\right)^{2}}{2 r_{0}^{2}}\right) d r=d_{0}^{2}+3 r_{0}^{2}
$$

Les distributions tracées sur la Figure $6 a$ ont été calculées pour plusieurs valeurs de $\mathrm{r}_{0}$, la valeur de $\mathrm{d}_{0}$ étant déduite de l'équation (30). Pour cette distribution, on obtient une forme analytique pour la largeur de la Lorentzienne : 


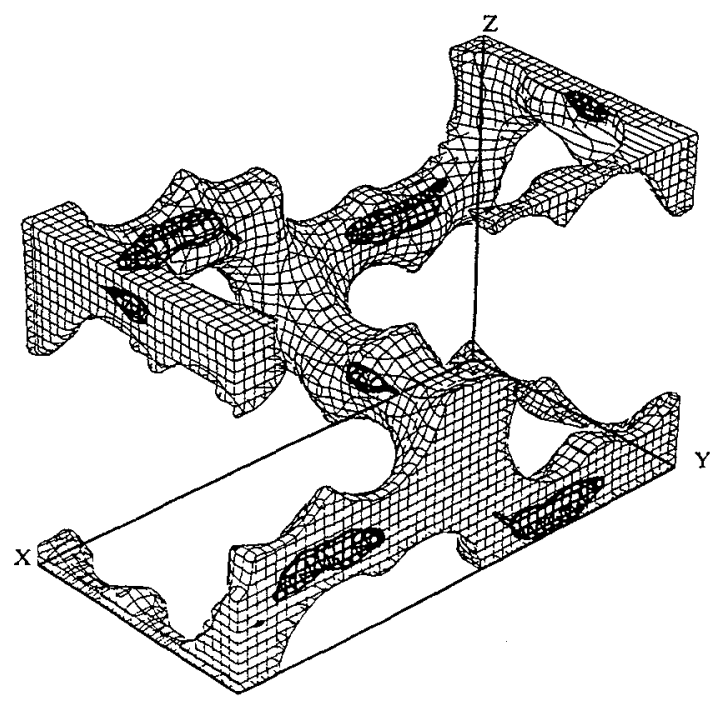

Figure 5. Distribution du centre de masse obtenu par simulation de type Monte Carlo pour du n-butane dans la silicalite à 400 K [14] ; les volumes plus foncés correspondent aux régions de la zéolithe où la probabilité de trouver le centre de masse de la molécule est supérieur à 50\%. Les lignes plus claires indiquent le volume libre de la zéolithe.
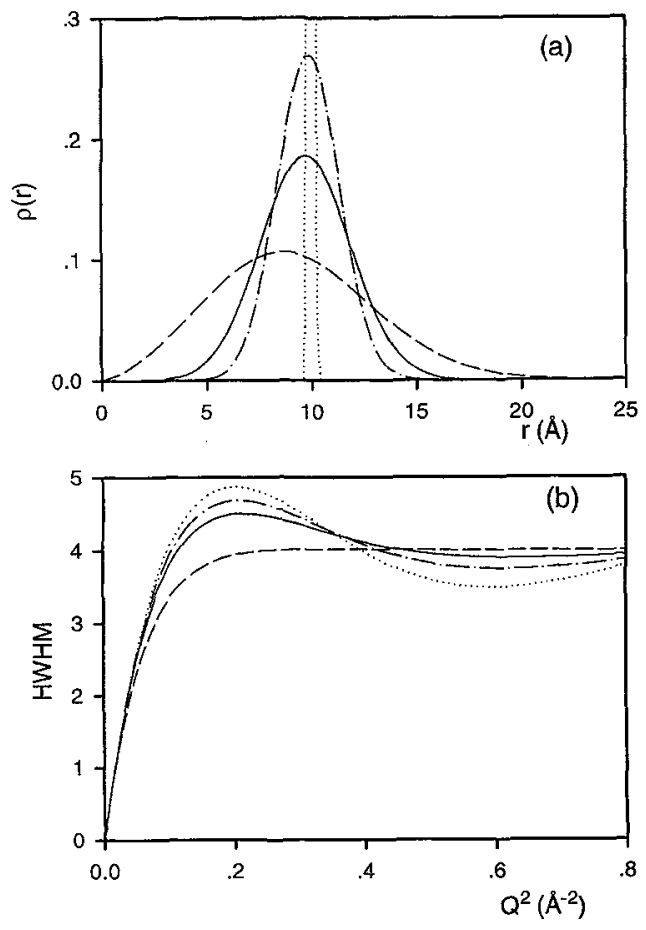

Figure 6. Résultats obtenus pour le nouveau modèle de diffusion par sauts : (a) distributions calculées pour une même valeur de $\left\langle r^{2}\right\rangle=100 \AA^{2}$; la courbe en pointillés correspond à $r_{0}=0,1 \AA\left(d_{0}=9,998 \AA\right)$, celle en tirets - points à $r_{0}=1,5 \AA\left(d_{0}=9,657\right.$ $\AA)$, la courbe continue à $r_{0}=2,2 \AA\left(d_{0}=9,246 \AA\right)$, et les tirets à $r_{0}=4,5 \AA\left(d_{0}=6,265 \AA\right)$, (b) élargissements calculés en fonction de $Q^{2}$, les courbes ont les mêmes valeurs des paramètres qu'en (a). 


$$
\begin{aligned}
\Delta \omega(Q) & =\frac{1}{\tau}\left[1-\frac{1}{Q d_{0} r_{0}(2 \pi)^{1 / 2}} \int_{0}^{\infty} \sin (Q r) \exp \left(-\frac{\left(r-d_{0}\right)^{2}}{2 r_{0}^{2}}\right) d r\right] \\
& =\frac{1}{\tau}\left[1-\frac{\sin \left(Q d_{0}\right)}{Q d_{0}} \exp \left(-\frac{Q^{2} r_{0}^{2}}{2}\right)\right]
\end{aligned}
$$

Les deux limites de cette équation, pour $\mathrm{r}_{0}$ petit ou grand, correspondent aux modèles de $\mathrm{CE}$ et de HR (Figure 6b). Pour une faible valeur de $\mathrm{r}_{0}(0,1 \AA)$, on obtient la même largeur qu'avec l'équation (16). Pour une plus grande valeur de $\mathrm{r}_{0}(4,5 \AA)$, on obtient une distribution proche de celle de HR et la variation de la largeur est similaire à celui reporté sur la Figure 1.

L'équation (31) a le comportement attendu pour les deux cas limites : petit et grand Q. Pour les grandes valeurs de $Q$, les élargissements tendent vers $1 / \tau$. Quand $Q$ est petit, un développement des fonctions sinus et exponentielle donne comme expression :

$$
\Delta \omega(Q)=\frac{1}{\tau}\left[1-\left(1-\frac{Q^{2} d_{0}^{2}}{6}\right)\left(1-\frac{Q^{2} r_{0}^{2}}{2}\right)\right]
$$

En négligeant le terme en $Q^{4}$ et en utilisant les équations (30) et (25) il vient

$$
\Delta \omega(Q)=\frac{Q^{2}}{6 \tau}\left(d_{0}^{2}+3 r_{0}^{2}\right)=D Q^{2}
$$

En conclusion, quel que soit le modèle, on doit pouvoir mesurer D. Par contre il sera plus difficile de déterminer la distribution de distances de sauts.

\section{DIFFUSION ANISOTROPE}

Différents modèles de diffusion dans des géométries restreintes ont déjà été examinés : diffusion entre deux plans [15], dans une sphère [16] et dans un cylindre [17]. Le cas de la diffusion anisotrope dans un milieu infini a été appliqué aux cristaux liquides, en introduisant deux coefficients de diffusion : l'un le long de l'axe principal de la molécule, l'autre perpendiculaire à cet axe [18].

Certains matériaux représentent des systèmes unidimensionnels (1D) parfaits, essentiellement les zéolithes et les aluminophosphates, et également peut-être les nouvelles silices mésoporeuses (de type $\mathrm{MCM}$ ) et les nanotubes de carbone. Dans ces matériaux, les canaux dont le diamètre varie de 4 à $40 \AA$ sont parallèles et non connectés. Chaque molécule reste en permanence dans le même canal, la longueur du canal pouvant aller jusqu'à plusieurs centaines de $\mu \mathrm{m}$.

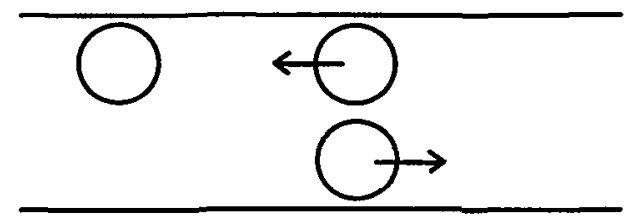

(a) Diffusion $1 \mathrm{D}$ normale

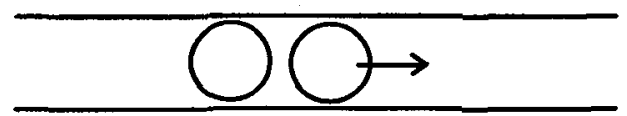

(b) Diffusion 1D à la file indienne

Figure 7. Deux cas différents de diffusion unidimensionnelle. 
Deux cas différents peuvent se produire : si les molécules dans un canal donné peuvent se croiser, on parle alors de diffusion 1D normale (Figure 7). Cependant, si le rayon du canal est inférieur au diamètre de la molécule, il n'y a pas de croisement possible, les molécules doivent garder le même ordre et ce cas spécial est appelé diffusion à la file indienne. Les implications de ce modèle ont été envisagées depuis longtemps dans les membranes biologiques [19], mais ce n'est que récemment que des mesures microscopiques par RMN en gradient de champ pulsé [20] et par DQEN [21] ont été réalisées.

Nous examinerons ces deux cas séparément, des exemples étant donnés dans la partie 5.3.

\subsection{Diffusion unidimensionnelle normale}

On considère une molécule qui diffuse dans un canal unidimensionnel, avec un coefficient de diffusion $\mathrm{D}$. On néglige tout mouvement perpendiculaire à l'axe du canal, ce qui est correct quand les molécules peuvent juste se croiser (Figure 7a), mais ce mouvement moyenne la fonction de diffusion si le diamètre du canal est plus important (exemple du méthane dans un nanotube de carbone).

En définissant par $\theta$ l'angle entre l'axe du canal et la direction de $\mathbf{Q}$, la fonction de diffusion incohérente (cf. équation 11) devient pour ce canal [22]

$$
S_{1 D}(\mathbf{Q}, \omega)=\frac{1}{\pi} \frac{D Q^{2} \cos ^{2} \theta}{\omega^{2}+\left(D Q^{2} \cos ^{2} \theta\right)^{2}}
$$

Le profil est toujours Lorentzien, mais la largeur dépend de l'angle $\theta$. Si l'on pouvait disposer d'un échantillon orienté, il serait alors possible d'obtenir un élargissement maximum des spectres en alignant le vecteur $\mathbf{Q}$ selon l'axe des canaux, et une largeur nulle (i.e. la résolution instrumentale) en orientant $\mathbf{Q}$ perpendiculaire à l'axe des canaux (Figure 8). En fait il s'est avéré impossible de pouvoir préparer en quantité suffisante des échantillons orientés de zéolithes. Les cristaux les plus gros ont comme dimension $400 \times 100 \times 100 \mu \mathrm{m}^{3}$, et il en faut des centaines de milliers pour atteindre $1 \mathrm{~g}$, la quantité minimum pour avoir un signal suffisant de l'adsorbat.

On est donc obligé d'utiliser des poudres, et il faut alors moyenner l'équation (34) :

$$
S_{1 D}(Q, \omega)=\frac{1}{2 \pi} \int_{0}^{\pi} d \theta \frac{D Q^{2} \cos ^{2} \theta \sin \theta}{\omega^{2}+\left(D Q^{2} \cos ^{2} \theta\right)^{2}}
$$

En intégrant cette équation on obtient :

avec

$$
S_{1 D}(Q, \omega)=\frac{1}{4 \pi \sqrt{2} \omega y}\left\{\ln \left(\frac{1+y^{2}-\sqrt{2} y}{1+y^{2}+\sqrt{2} y}\right)+2 \arctan (1+\sqrt{2} y)-2 \arctan (1-\sqrt{2} y)\right\}
$$

$$
y^{2}=\frac{D Q^{2}}{\omega}
$$

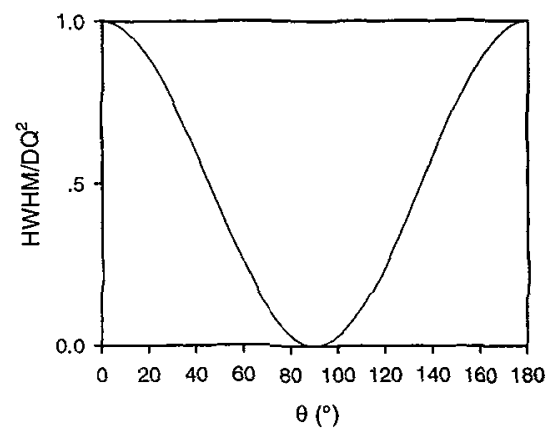

Figure 8. Dépendance de la largeur du modèle de diffusion unidimensionnelle normale en fonction de l'angle entre le vecteur Q et l'axe du canal. 
11 est intéressant de noter que l'on aboutit à la même expression en calculant la fonction de diffusion pour un échantillon isotrope de canaux 1D [23]. En effet, la fonction d'autocorrélation pour un canal donné est toujours une Gaussienne mais de la forme

$$
G_{1}(r, t)=\frac{1}{\sqrt{2 \pi<\sigma^{2}>}} \exp \left(-\frac{r^{2}}{2<\sigma^{2}>}\right)
$$

Où

$$
\left.<\sigma^{2}\right\rangle=2 D t
$$

est le déplacement carré de la particule pour une diffusion à une dimension (comparer à l'équation 25). Pour passer à la fonction d'autocorrélation en trois dimensions, à partir de celle en une dimension, on tient compte du fait que la probabilité de trouver un canal donné sur la surface d'une sphère de rayon $r$ est $1 / 2 \pi r^{2}$ (il y a un facteur 2 par rapport à la surface de la sphère car un canal donné localisé sur la surface en $\mathbf{r}$ est aussi trouvé en -r)

$$
G_{3}(\mathbf{r}, t)=\frac{1}{2 \pi r^{2}} G_{1}(r, t)=\frac{1}{(2 \pi)^{3 / 2}(2 D t)^{1 / 2} r^{2}} \exp \left(-\frac{r^{2}}{4 D t}\right)
$$

Alors qu'en 3D, la fonction d'autocorrélation était une Gaussienne (équation 7), l'équation (39) $n$ 'en est plus une. Le facteur $r^{2}$ introduit une discontinuité pour $r=0$. Ainsi, la fonction d'autocorrélation pour une diffusion $1 D$ est plus piquée autour de l'origine. La fonction de diffusion est la transformée de Fourier sur $\mathbf{r}$ et sur $t$ de l'équation (39):
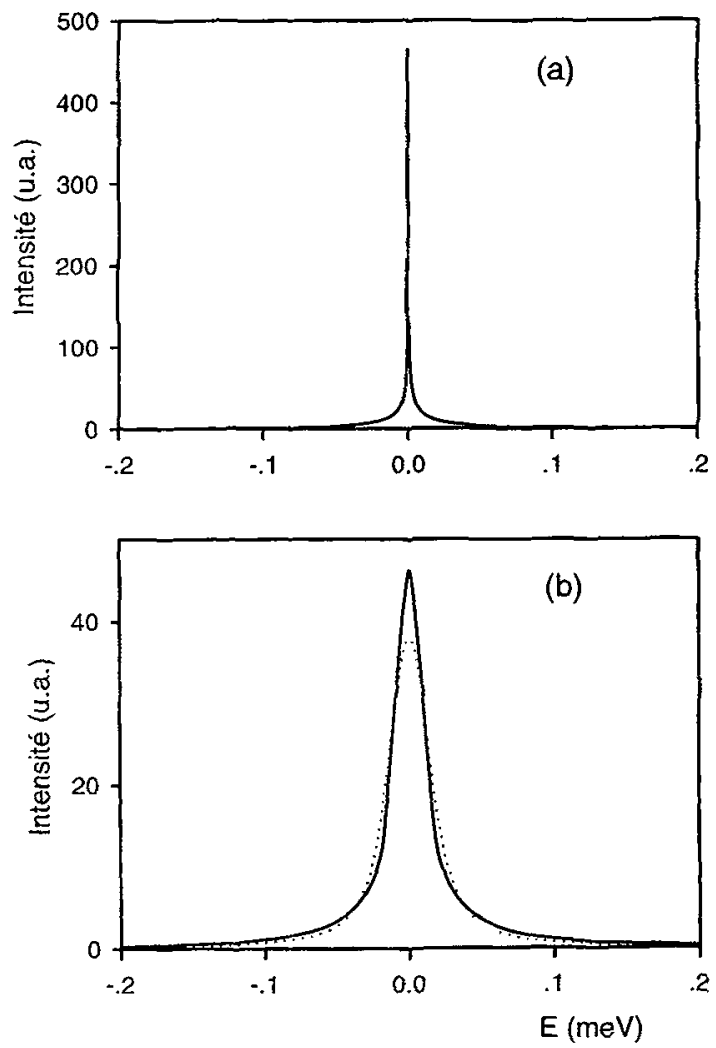

Figure 9. Spectres simulés pour un modèle de diffusion $1 \mathrm{D}$ normale $\left(\mathrm{D}=10^{-8} \mathrm{~m}^{2} \mathrm{~s}^{-1}, \mathrm{Q}=0,3 \AA^{-1}\right)$. La fonction de diffusion est convoluée avec une résolution en forme de triangle de différente largeut totale à mi-hauteur (a) $0,2 \mu \mathrm{eV}$, (b) $18 \mu \mathrm{eV}$, La ligne en pointillés dans la figure (b) correspond à une diffusion 3D. 


$$
S_{1 D}(\mathbf{Q}, \omega)=\frac{1}{2 \pi} \int d t \int d \mathbf{r} \exp (-i \omega t) \exp (i \mathbf{Q} \cdot \mathbf{r}) G_{3}(\mathbf{r}, t)
$$

Après intégration, on retombe sur l'expression (36). Ainsi, quel que soit l'ordre d'intégration, $S_{1 D}$ diverge pour $\omega=0$. Ceci peut être relié à la discontinuité de $G_{3}(r, t)$. Expérimentalement, on n'observe pas de discontinuité dans les spectres à cause de la résolution de l'instrument qui est finie. Pour l'interprétation des spectres, il est plus facile de convoluer l'équation (34) avec la résolution instrumentale et de calculer ensuite la moyenne de poudre numériquement.

Pour ce modèle de diffusion, on ne peut plus raisonner en terme de largeur à mi-hauteur en fonction de $Q^{2}$ car le profil des spectres n'est plus du tout Lorentzien. Pour mettre cet effet en évidence, il faut évidemment que la résolution de l'instrument soit suffisante. Des spectres calculés pour deux résolutions très différentes sont présentés sur la Figure 9 . La valeur de $Q$ est de $0,3 \AA^{-1}$, et celle choisie pour $\mathrm{D}$ de $10^{-8} \mathrm{~m}^{2} \mathrm{~s}^{-1}$, ce qui correspond à du méthane diffusant dans une zéolithe à canaux. Dans la Figure 9(a) la résolution est un triangle (FWHM : 0,2 $\mu \mathrm{eV}$ ) ; dans la Figure 9(b) c'est aussi un triangle, mais la largeur totale à mi-hauteur est de $18 \mu \mathrm{eV}$ (conditions classiques sur IN5 à l'ILL). La ligne en pointillé sur la Figure 9(b) correspond au profil que l'on attendrait si la diffusion était en 3D. Une Lorentzienne, convoluée par la résolution instrumentale, a été ajustée sur le spectre calculé pour la diffusion 1D. Avec une résolution de $18 \mu \mathrm{eV}$, et pour les valeurs des paramètres choisis, on peut ainsi distinguer entre une diffusion $1 \mathrm{D}$ et $3 \mathrm{D}$, mais la statistique doit être suffisante.

\subsection{Diffusion unidimensionnelle à la file indienne}

Comme pour le modèle de diffusion 1D normale, on peut calculer la fonction de diffusion pour une diffusion $1 \mathrm{D}$ à la file indienne de deux façons : soit en dérivant la fonction de diffusion pour un canal et en prenant la moyenne de poudre, soit en considérant une distribution isotrope de canaux et en calculant la fonction de diffusion à partir de la fonction d'autocorrélation tridimensionnelle [23]. Nous exposerons ici la première façon. La fonction d'autocorrélation pour un canal a la même expression que dans l'équation (37) mais le déplacement carré moyen pour une diffusion à la file indienne a pour valeur [24]

$$
<\sigma^{2}>=2 F t^{1 / 2}
$$

La principale différence avec la diffusion 1D normale est que le déplacement carré moyen n'est plus proportionnel au temps mais à la racine carrée du temps. A cause des corrélations entre les molécules, la diffusion à longue distance est ainsi réduite. Le pendant du coefficient de diffusion $\mathrm{D}$ est le facteur de mobilité $\mathrm{F}$, il a comme unité des $\mathrm{m}^{2} \mathrm{~s}^{-1 / 2}$. En prenant dans un premier temps l'axe du canal parallèle à la direction de $\mathbf{Q}$, on obtient pour la fonction de diffusion intermédiaire

$$
I(Q, t)=\int d r G_{1}(r, t) \exp (i Q r)=\exp \left(-F Q^{2} t^{1 / 2}\right)
$$

Par rapport à la diffusion normale (3D ou 1D), le temps est à la puissance $1 / 2$. La transformée de Fourier par rapport au temps donne pour la fonction de diffusion

$$
\begin{aligned}
S_{S F}(Q, \omega) & =\frac{1}{\pi} \int_{0}^{\infty} d t \cos (\omega t) \exp \left(-F Q^{2} t^{1 / 2}\right) \\
& =\frac{z}{\omega}\left[\cos \left(\frac{\pi}{2} z^{2}\right)\left(\frac{1}{2}-C(z)\right)+\sin \left(\frac{\pi}{2} z^{2}\right)\left(\frac{1}{2}-S(z)\right)\right]
\end{aligned}
$$

avec

$$
z^{2}=\frac{F^{2} Q^{4}}{2 \pi \omega}
$$

$C(v)$ et $S(v)$ représentent les intégrales de Fresnel. Pour un échantillon sous forme de poudre, il faut prendre la moyenne :

$$
S_{S F}(Q, \omega)=\frac{F Q^{2}}{\sqrt{\pi}(2 \omega)^{3 / 2}} \int_{0}^{\pi} d \theta \sin \theta \cos ^{2} \theta\left[\cos \left(\frac{\pi}{2} z^{2}\right)\left(\frac{1}{2}-C(z)\right)+\sin \left(\frac{\pi}{2} z^{2}\right)\left(\frac{1}{2}-S(z)\right)\right]
$$

Pour le calcul numérique de cette fonction, il est utile d'introduire la fonction auxiliaire [25] 


$$
g(z)=\left[\cos \left(\frac{\pi}{2} z^{2}\right)\left(\frac{1}{2}-C(z)\right)+\sin \left(\frac{\pi}{2} z^{2}\right)\left(\frac{1}{2}-S(z)\right)\right]
$$

On peut prendre comme approximation [25]

Où

$$
g(z)=\frac{1}{2+4,142 z+3,492 z^{2}+6,670 z^{3}}+\varepsilon(z)
$$

Cette approximation est valable dans les ailes mais quand $\omega$ est très proche de zéro, il est préférable de calculer directement les intégrales de Fresnel.

Deux profils calculés pour ce modèle, avec une résolution en forme de triangle, sont représentés sur la Figure 10. La valeur de Q est de $0,3 \AA^{-1}$ et celle du facteur de mobilité $\mathrm{F}$ de $10^{-11} \mathrm{~m}^{2} \mathrm{~s}^{-1 / 2}$. Le spectre calculé avec une très bonne résolution ( $F W H M=0,2 \mu \mathrm{eV}$ ) est encore plus étroit que dans le cas de la diffusion 1D normale. Avec une résolution plus classique sur IN5 (FWHM $=18 \mu \mathrm{eV})$, le profil reste étroit et ne peut pas être ajusté par une diffusion 3D (ligne pointillée sur la Figure 10b).
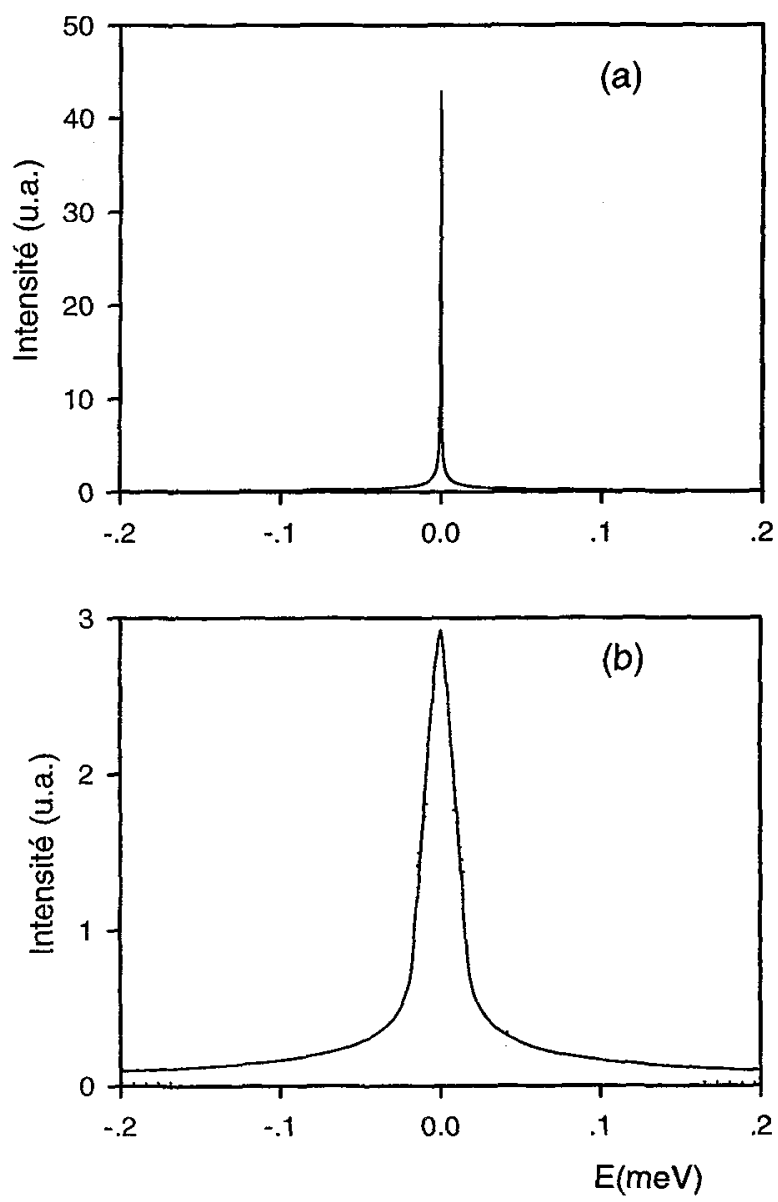

Figure 10. Spectres simulés pour un modèle de diffusion $1 D$ à la file indienne $\left(F=10^{-11} \mathrm{~m}^{2} \mathrm{~s}^{-1 / 2}, Q=0,3 \AA^{-1}\right)$. La fonction de diffusion est convoluée avec une résolution en forme de triangle de différente largeur totale à mi-hauteur (a) $0,2 \mu \mathrm{eV}$, (b) 18 $\mu \mathrm{VV}$. La ligne en pointillés dans la figure (b) correspond à une diffusion 3D. 


\section{APPLICATION AUX MOLECULES ADSORBEES DANS LES ZEOLITHES}

Les zéolithes sont des matériaux microporeux bien cristallisés d'origine naturelle ou synthétique (au départ il ne s'agissait que d'aluminosilicates). Leur formule chimique est de la forme $\mathrm{M}_{\mathrm{x} / \mathrm{n}}\left[\left(\mathrm{AlO}_{2}\right)_{\mathrm{x}}\left(\mathrm{SiO}_{2}\right)_{\mathrm{y}}\right.$ ]॰ $m \mathrm{H}_{2} \mathrm{O}$, où $\mathrm{M}$ est un cation de valence $\mathrm{n}$ et $m$ le nombre de molécules d'eau dans la maille. La charpente est constituée d'un réseau tridimensionnel de tétraèdres $\mathrm{SiO}_{4}$ et $\mathrm{AlO}_{4}$ liés entre eux par les atomes d'oxygène. Une fois déshydratées, le volume interne constitué de cavités ou de canaux peut être occupé par des molécules dont la nature dépendra de leur taille et de leur force d'interaction avec les cations ou avec la charpente de la zéolithe. Comme les dimensions des pores zéolithiques sont comprises entre 3 et $12 \AA$, on utilise le terme de tamis moléculaire pour désigner ces matériaux.

Depuis les années 80 , d'autres familles de matériaux microporeux, tels que les aluminophosphates de type $\mathrm{AlPO}_{4}$ sont apparues. Cependant, bien qu'il existe plus d'une centaine de structures différentes de zéolithes ou de matériaux microporeux apparentés, seul un petit nombre est utilisé à grande échelle dans l'industrie. On peut mentionner leur application comme catalyseurs dans le raffinage et la pétrochimie, comme échangeurs d'ions (dans les lessives à la place des phosphates), comme adsorbants (par exemple dans les doubles vitrages), dans le domaine agricole, etc.

De multiples techniques physiques sont nécessaires pour élucider les phénomènes complexes mis en jeu dans les zéolithes: la diffraction (rayons $\mathrm{X}$ et neutron) permet de déterminer la structure et éventuellement les sites occupés par les molécules [26]. Les méthodes spectroscopiques donnent des informations sur les propriétés de la zéolithe et sur l'interaction entre la molécule et la charpente [27]. Des techniques plus spécifiques ont été mises en œuvre pour étudier la diffusion [28]. Les différentes méthodes théoriques interviennent maintenant à toutes les étapes de l'interprétation: structure, adsorption, réaction, et diffusion.

Il est pratique de scinder en deux blocs les techniques expérimentales qui permettent d'étudier la diffusion dans les zéolithes: les méthodes macroscopiques et les méthodes microscopiques. Les méthodes macroscopiques telles que la gravimétrie, la volumétrie, la chromatographie, etc. fonctionnent dans des conditions hors équilibre. On obtient des coefficients de diffusion en présence d'un gradient de concentration et les résultats obtenus sont dénommés diffusivités de transport. Au contraire, les mesures 'microscopiques' sont réalisées à l'équilibre, sur des échantillons isolés. Les méthodes opérant dans ces
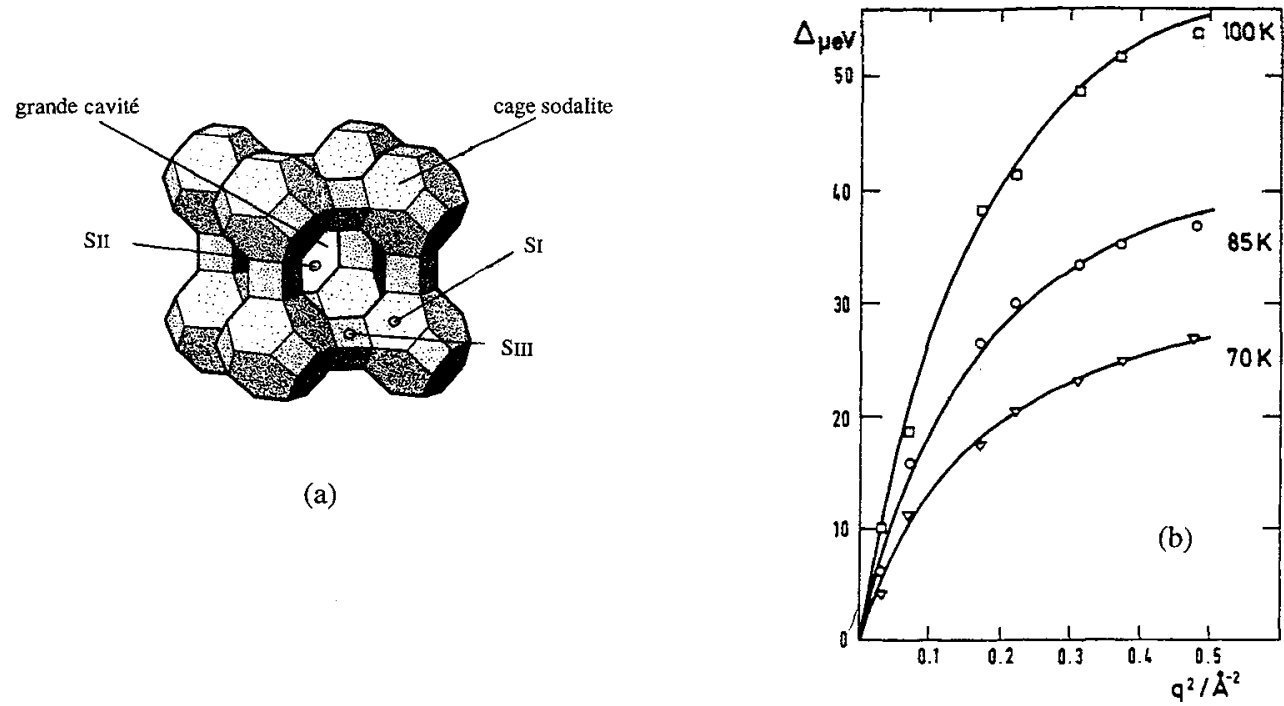

Figure 11. (a) Structure de la zéolithe NaA; (b) Elargissements mesurés à différentes températures pour 3,4 molécules d'hydrogène adsorbées dans la zéolithe $\mathrm{NaA}[30]$. Les symboles $(\square, O, \dot{\nabla})$ sont obtenus à partir de fits individuels des spectres, les courbes en continu correspondent au modèle SS. 
conditions sont la DQEN et la RMN en gradient de champ pulsé (PFG NMR), le coefficient de diffusion. obtenu étant le coefficient d'autodiffusion.

Parmi les nombreux exemples qui sont maintenant disponibles en DQEN, nous en examinerons trois qui sont représentatifs des molécules et des structures zéolithiques étudiées.

\subsection{Hydrogène dans les zéolithes $\mathrm{NaA}$ et $\mathrm{NaX}$}

La diffusion de l'atome d'hydrogène dans les hydrures a été évoquée dans la partie 3.2.1. Dans une zéolithe, l'hydrogène que l'on adsorbe reste sous forme moléculaire. Cette molécule a un intérêt pour un certain nombre de procédés industriels, par exemple les réactions d'hydrogénation avec des réacteurs à membrane, dans lesquels la zéolithe constitue la membrane poreuse [29]. De part sa petite taille et son interaction relativement faible avec une zéolithe, on s'attendrait à ce que la diffusion de l'hydrogène soit rapide et peu sensible à la structure de la zéolithe. En réalité, des expériences récentes montrent que la situation est plus compliquée que prévue.

Aucune mesure macroscopique concernant l'hydrogène dans les zéolithes n'a été réalisée jusqu'à présent. La DQEN a permis de mesurer la diffusion à longue distance dans la NaA [30] et la NaX [4] ; dans la chabazite [31] et la mordénite [32] seuls des mouvements locaux ont semble-t-il été observés. Des résultats récents viennent d'être obtenus par PFG NMR [33].

La structure de la zéolithe $\mathrm{NaA}$, de composition $\mathrm{Na}_{12}\left[\left(\mathrm{AlO}_{2}\right)\left(\mathrm{SiO}_{2}\right)\right]_{12}$, est montrée de façon schématique sur la Figure 11(a). Les lignes qui sont tracées joignent les atomes de silicium ou d'aluminium (on peut parfois différencier ces deux atomes par diffraction), les atomes d'oxygène ne sont pas représentés, ils se situent au milieu des liaisons Si-Al. Les petites cavités sodalite ne sont accessibles que par de petites molécules telles que l'eau (on ignore si l'hydrogène s'adsorbe à l'intérieur de ces cavités). L'assemblage des cages sodalite tel qu'il est représenté sur la Figure 11(a) constitue un réseau cubique simple de cavités plus importantes, dont le diamètre libre est $\approx 11,4 \AA$. Les grandes cavités sont reliées entre elles par des fenêtres dont le diamètre est $\approx 4,2 \AA$, ce diamètre pouvant être réduit du fait de la présence de cations sodium en position SII. Le nombre et la position des cations ont un effet important sur les propriétés de diffusion. Dans une pseudo maille, on situe de façon idéale $8 \mathrm{Na}$ en site SI, 3 en site SII près du centre de la fenêtre (comme une fenêtre est partagée entre deux cavités, on trouve un sodium
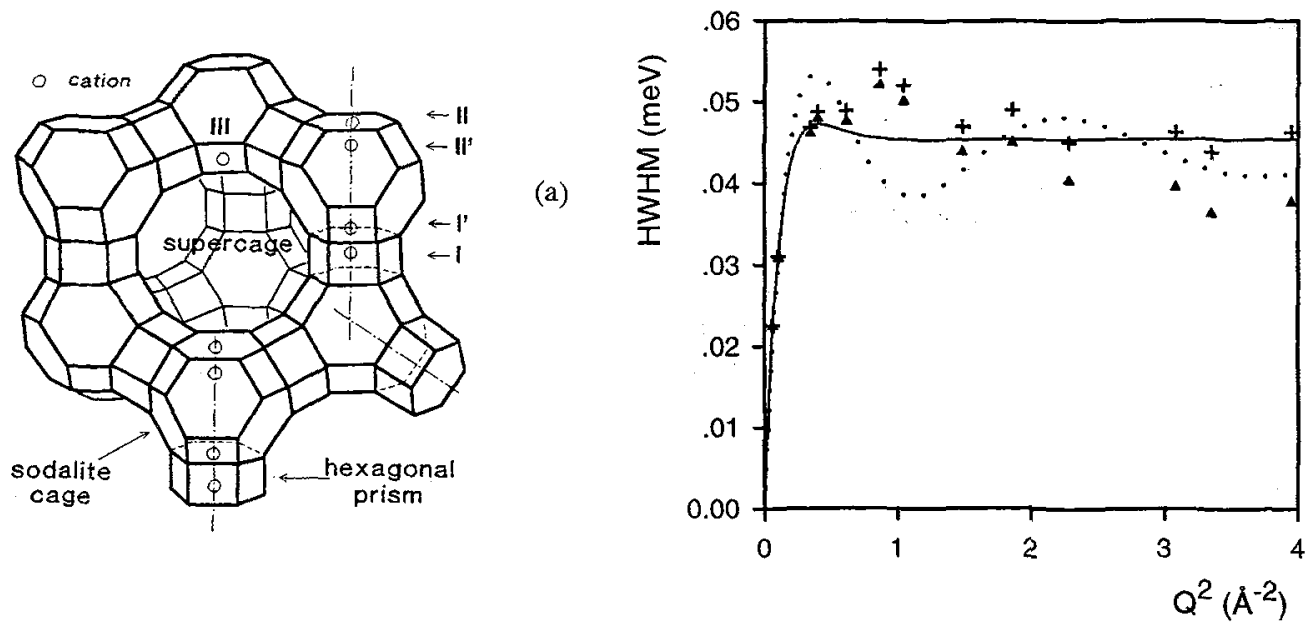

Figure 12. (a) Structure de la zéolithe NaX ; (b) Elargissements mesurés pour 4 molécules d'hydrogène adsorbées à $100 \mathrm{~K}$ dans la zéolithe $\mathrm{NaX}$. Les croix et les triangles sont obtenus à partir des fits individuels des spectres (voir texte). Les courbes correspondent à des fits de tous les spectres en même temps : celle en pointillés au modèle CE et celle en continu au nouveau modèle. 
dans chaque fenêtre), et $1 \mathrm{Na}$ dans un site SIII (il y a 12 sites SIII équivalents dans une grande cavité). En échangeant des cations sodium par des cations divalents, tel que le calcium, on diminue le nombre de cations dans les fenêtres et on arrive ainsi à agrandir le diamètre des pores. Cependant, seuls les gaz rares, $\mathrm{O}_{2}, \mathrm{~N}_{2}, \mathrm{H}_{2} \mathrm{O}$ ou les alcanes linéaires peuvent être adsorbés dans cette structure.

En DQEN, pour concentrer un nombre suffisant de molécules d'hydrogène dans le faisceau, on doit réaliser les expériences à basse température. II faut cependant tenir compte du fait que le rapport ortho/para $\mathrm{H}_{2}$ diminue avec la température, le para $\mathrm{H}_{2}$ diffusant beaucoup moins les neutrons de faible énergie. Avec $\mathrm{H}_{2}$, les expériences ont été réalisées entre 70 et $150 \mathrm{~K}[30,4]$. Pour pouvoir faire des mesures au-dessous de $20 \mathrm{~K}$, on peut utiliser la molécule HD [34], étant donné que la séparation entre niveaux de rotation symétriques et antisymétriques n'existe plus.

Dans la NaA, la variation de la largeur en fonction de $\mathrm{Q}^{2}$ (Figure $11 \mathrm{~b}$ ) a été ajustée au moyen du modèle SS, la distance de saut moyenne étant de 3,9 $\AA$ [30]. Pour des taux de remplissage de 1,25 et 1,85 molécules par cavité, il a été trouvé que le coefficient de diffusion restait constant, $D=5,1 \times 10^{-5} \mathrm{~cm}^{2} \mathrm{~s}^{-1}$; par contre une faible augmentation de ce coefficient (de 20\%) a été constatée pour un remplissage de 3,4 molécules par cavité. L'énergie d'activation pour la diffusion est relativement faible : $2 \mathrm{~kJ} \mathrm{~mol}^{-1}$.

Les zéolithes synthétiques $\mathrm{X}$ et $\mathrm{Y}$ ont la même structure (Figure 12a) que la faujasite, minéral naturel. Les zéolithes de type $\mathrm{X}$ ont un rapport $\mathrm{Si} / \mathrm{Al}$ plus faible $(1,0-1,5)$ que la $\mathrm{Y}(1,5-3,0)$, elles ont ainsi plus de cations de compensation. La supercage a un diamètre de $\approx 12,5 \AA$ et les fenêtres entre deux supercages sont bien plus larges que pour la zéolithe $A: \approx 7,5 \AA$. Grâce aux vibrations de la charpente, des molécules d'un diamètre cinétique jusqu'à $10 \AA$ arrivent à pénétrer dans le réseau (le diamètre cinétique est la distance intermoléculaire la plus proche pour 2 molécules qui se rencontrent avec une énergie cinétique nulle, il est plus petit que la distance d'équilibre). L'espace accessible dans cette structure est de $50 \%$ en volume. Dans la $\mathrm{NaX}$, de composition $\mathrm{Na}_{86} \mathrm{Al}_{86} \mathrm{Si}_{106} \mathrm{O}_{384}$, la distribution cationique est plus compliquée que dans la $\mathrm{NaA}$ : on a répertorié quatre types de sites occupés : I, I', II et III. Les sites SI et SI' sont partiellement occupés (étant donné que leur distance est seulement de $2,18 \AA$, ils ne peuvent pas être occupés simultanément). Les sites SI, quatre par supercage, sont tous occupés mais seulement la moitié environ des sites SIII sont occupés. Pour ces raisons, il est pratiquement impossible de faire des simulations détaillées sur une zéolithe de cette composition.

Des expériences DQEN ont été réalisées à $100 \mathrm{~K}$, pour des taux de remplissage allant de 0,8 à 7,5 molécules par supercage, en moyenne [4]. Les spectres individuels ont été ajustés au moyen de deux modèles : le premier correspond simplement à un mouvement de diffusion à longue distance (les croix sur la Figure 12b), le second considère un mouvement de diffusion convolué par un mouvement de rotation isotrope (les triangles sur la Figure 12b). Comme le rayon de giration pour la molécule d'hydrogène est très petit, $0,37 \AA$, l'influence de la rotation est négligeable en-dessous de $1 \AA^{-1}$. En fittant tous les spectres en même temps avec le modèle $\mathrm{CE}$ isotrope (équation 16), on obtient la courbe en pointillés sur la Figure 12(b). L'accord est satisfaisant aux petites valeurs de $Q$ mais on voit que le modèle a des oscillations trop fortes à grand $\mathrm{Q}$. Pour avoir une information globale sur la valeur de l'affinement, on peut calculer comme en diffraction de poudre le facteur de confiance relatif au profil :

$$
R_{w p}=\left(\frac{\sum_{i} w_{i}\left[y_{i}(o b s)-y_{i}(c a l c)\right]^{2}}{\sum_{i} w_{i}\left[y_{i}(o b s)\right]^{2}}\right)^{1 / 2}
$$

on a utilisé comme poids $\quad w_{i}=\frac{1}{y_{i}(o b s)}$

Avec le modèle $C E$, on obtient $R_{w p}=11,7 \%$. En utilisant le nouveau modèle, équation (31), on améliore l'affinement, $R_{w p}=10,97 \%$, mais on n'arrive pas à reproduire fidèlement l'élargissement déterminé pour chacun des spectres individuels. Il est probable qu'en raison de la distribution complexe des cations, on ne puisse pas affiner l'ensemble des spectres avec une seule espèce d'hydrogène. Il serait intéressant de faire des mesures dans la $\mathrm{NaY}$, car cette structure ne contient pas de sodium en SIII, seulement en SII. 
Une description plus fine du mécanisme de saut nécessiterait les positions d'équilibre des molécules d'hydrogène. Malgré tout, les coefficients de diffusion obtenus à partir des petites valeurs de $\mathrm{Q}$ sont correctes et en bon accord avec les résultats de PFG NMR [33]. Avec les deux techniques, on observe que le coefficient de diffusion augmente quand la concentration en $\mathrm{H}_{2}$ augmente. Par DQEN, on trouve que D augmente d'un facteur 2 quand le taux de remplissage passe de 0,8 à 7,5 molécules par maille, de $3,75 \times 10^{-5} \mathrm{~cm}^{2} \mathrm{~s}^{-1}$ à $7,42 \times 10^{-5} \mathrm{~cm}^{2} \mathrm{~s}^{-1}$. Ceci indique que les molécules d'hydrogène sont en interaction avec les cations sodium, les premières molécules étant plus fortement adsorbées. A plus forte concentration, le temps de résidence obtenu par DQEN diminue, ce qui va bien dans le sens d'une plus faible interaction avec les cations.

Si l'on compare les élargissements des Figures 11(b) et 12(b), on constate que la tendance est similaire aux petites valeurs de $Q$, mais il est possible que la valeur de $\mathrm{Q}^{2}$ max pour les expériences décrites en réf. $30: 0,47 \AA^{-2}$, soit trop faible pour pouvoir observer un éventuel rétrécissement à grand $Q$.

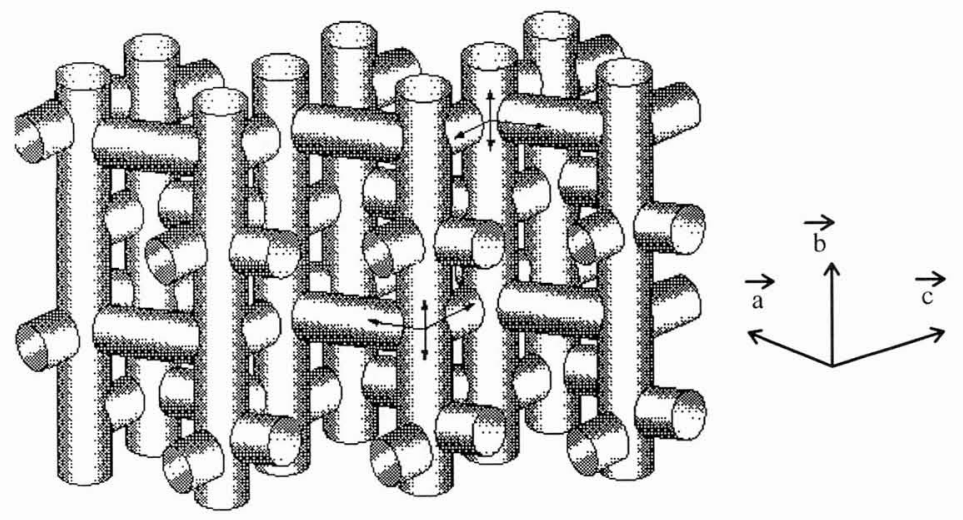

Figure 13. Représentation schématique des canaux dans la structure MFI (zéolithes ZSM-5 ou silicalite).

\subsection{Alcanes dans la structure MFI (ZSM-5 ou silicalite)}

Les deux zéolithes précédentes, $\mathrm{NaA}$ et $\mathrm{NaX}$, ont un réseau cubique et par conséquent les problèmes de moyenne de poudre présentés dans la partie 3.2.1 ne se posaient pas, du moins pour des valeurs de $Q$ inférieures à $2 \AA^{-1}$. La situation est plus compliquée dans le cas de la structure MFI (Figure 13). Ce code à trois lettres correspond aux zéolithes ZSM-5 et silicalite. La structure de ces deux zéolithes est la même, seul diffère leur rapport Si/Al qui est compris entre 12 et 50 pour la ZSM-5 et qui dépasse 1000 pour la silicalite. Ces deux zéolithes ont déjà de nombreuses applications en catalyse ou en adsorption. On s'active actuellement dans les laboratoires à préparer des membranes microporeuses dont la couche active est constituée d'un film mince de structure MFI. Ces membranes zéolithiques ont un potentiel intéressant dans le domaine de la séparation des gaz ou des hydrocarbures. D'autre part la silicalite, qui ne comporte pas de cations, est un cas idéal pour les calculs de simulation (Figure 5 par exemple).

Cette structure contient 2 types de canaux : des canaux droits parallèles à l'axe b et des canaux en zig-zag contenus dans le plan $(\mathrm{a}, \mathrm{c})$. Les sections des deux canaux sont des anneaux de 10 atomes d'oxygène, ce qui leur donne une taille de pore $(\approx 6 \AA$ ) intermédiaire entre la zéolithe $\mathrm{A}$ (anneaux à 8 oxygènes) et la faujasite (anneaux à 12 oxygènés).

A cause de son importance, la diffusion d'hydrocarbures dans cette structure a été étudiée en détail. Les coefficients de diffusion obtenus à $300 \mathrm{~K}$ par différentes techniques sont représentés sur la Figure 14 pour des alcanes linéaires courts $\left(<\mathrm{C}_{6}\right)$. Cet exemple illustre bien le problème de déterminer des coefficients de diffusion dans les zéolithes : les résultats s'étalent sur plusieurs ordres de grandeur. 


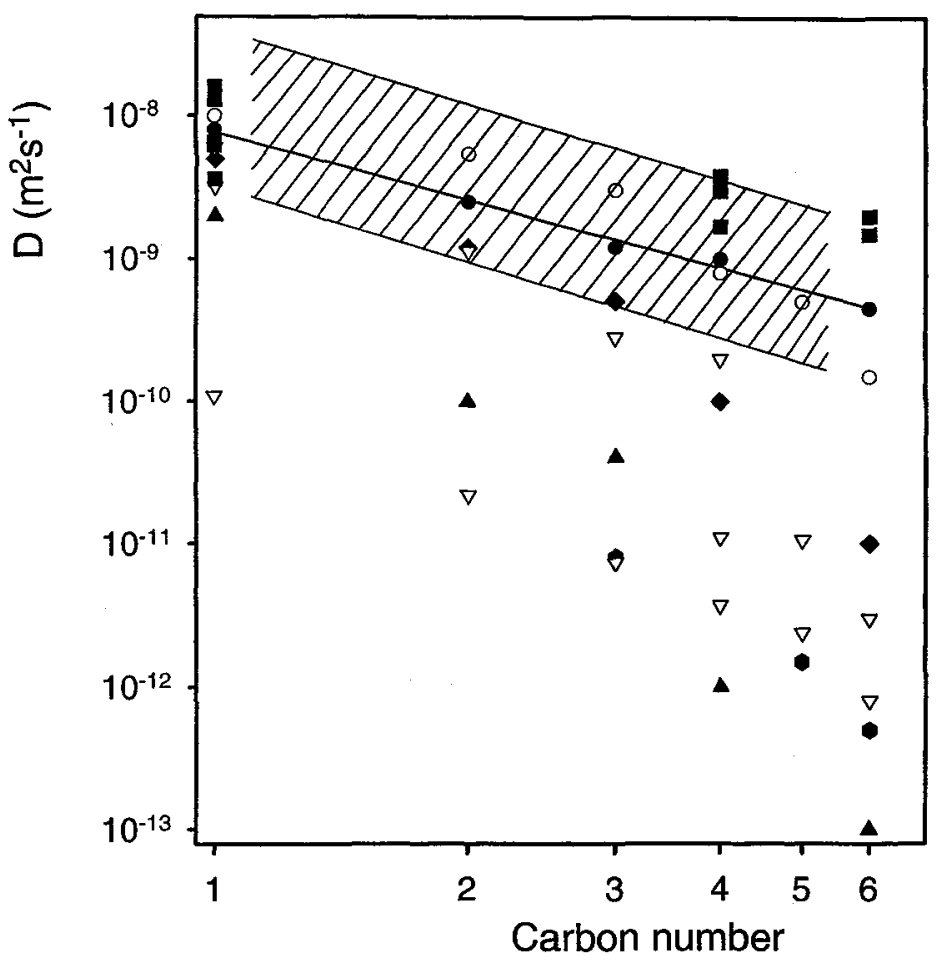

Figure 14. Coefficients de diffusion obtenus à $300 \mathrm{~K}$ pour différents alcanes dans les zéolithes ZSM-5 ou silicalite par plusieurs techniques: $(\bullet)$ DQEN [35-37], (O) PFG NMR [38,39], (五) simulations de dynamique moléculaire [40-49], ( $)$ réponse en fréquence [50,51], ( $\nabla)$ 'membrane' [52-54], (४) chromatographie [55,56], et (ه) ZLC [57].

Cependant, dans la zone hachurée, on trouve les valeurs obtenues par DQEN, par PFG NMR, et par dynamique moléculaire. Les résultats expérimentaux obtenus sur des poudres ont été comparés au coefficient de diffusion théorique moyen (le tiers de la trace du tenseur de diffusion). Pour certaines techniques, essentiellement macroscopiques, les désaccords énormes qui sont constatés indiquent que le transport de matière, tel qu'il est mesuré par ces méthodes, n'est pas exclusivement limité par la diffusion intracristalline (à l'intérieur des cristaux).

En DQEN, on peut se poser la question de l'influence de l'anisotropie du mouvement sur la précision des coefficients de diffusion obtenus. Pour tester cet effet, on a calculé des spectres pour les modèles de diffusion isotrope ou anisotrope. Pour la diffusion anisotrope, on a choisi un rapport d'anisotropie voisin de celui calculé par des simulations de dynamique moléculaire dans la silicalite $[41,43]: \mathrm{D}_{\|} / \mathrm{D}_{\perp}=5$, ce qui correspond à des molécules diffusant 5 fois plus vite le long des canaux droits que dans les canaux en zig-zag (Figure 13). Ce rapport de 5 a été obtenu pour l'hexane, il est même plus petit pour les alcanes plus courts $[41,43]$, ce qui signifie que l'anisotropie est moins importante. Quand les élargissements sont faibles (Figure 15), ce qui est généralement le cas aux petites valeurs de $Q$, on n'observe que de faibles différences entre les largeurs obtenues par les deux modèles ( $5 \%$ ), pour un même 

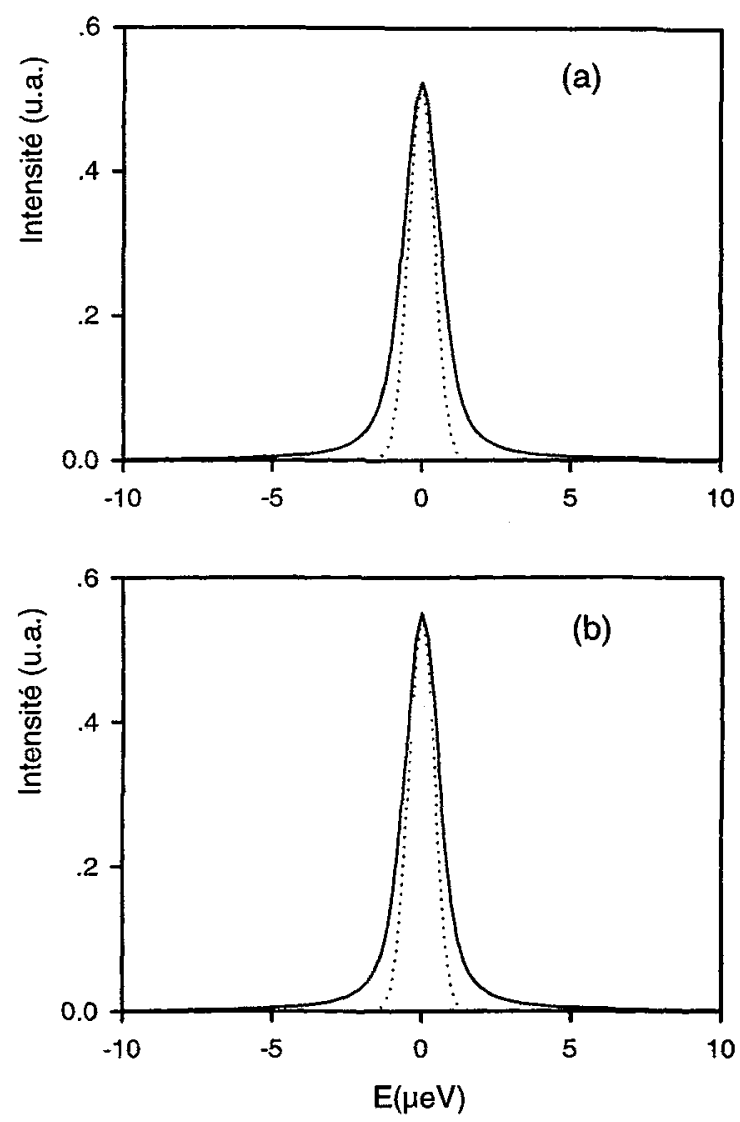

Figure 15. Spectres simulés pour une diffusion (a) isotrope, et (b) anisotrope. Le coefficient de diffusion moyen est le même. La fonction de diffusion est convoluée par une fonction de résolution en forme de Gaussienne, ligne en pointillés (FWHM = 1 $\mu \mathrm{eV})$.

coefficient de diffusion moyen. Cette variation est largement contenu dans la marge d'erreur habituellement rapportée pour les coefficients de diffusion $: \approx 50 \%$. Par contre, les élargissements peuvent être plus importants à grand $\mathrm{Q}$ et la différence entre les largeurs obtenues pour les deux modèles est alors de 30\% (Figure 16). Dans ce cas, on est en mesure de distinguer les 2 modèles, et si la statistique est suffisante de déterminer le rapport d'anisotropie [58].

Les spectres DQEN qui ont été obtenus dans cette structure sont plus proche de la Figure 15 que de la Figure 16 [35-37]. Les coefficients de diffusion moyens qui ont été déterminés avec un modèle de diffusion isotrope sont donc corrects, dans la limite de l'erreur expérimentale généralement admise. Cette approximation est connue depuis longtemps [22,59], mais apparemment pas par tout le monde [60]. Le même problème de moyenne se pose d'ailleurs en PFG NMR [61]. Il faut aussi garder à l'esprit que l'on raisonne sur des différences entre les techniques de plusieurs ordres de grandeur (Figure 14) et que l'on ne chipote pas pour des différences de $50 \%$ sur les coefficients de diffusion.

La diffusion des alcanes légers a pu être mesurée sur IN5 à l'ILL, l'interprétation des spectres faisant intervenir l'élargissement dû à la rotation. La diffusion d'alcanes linéaires plus longs, $\mathrm{C}_{8}-\mathrm{C}_{14}$, 

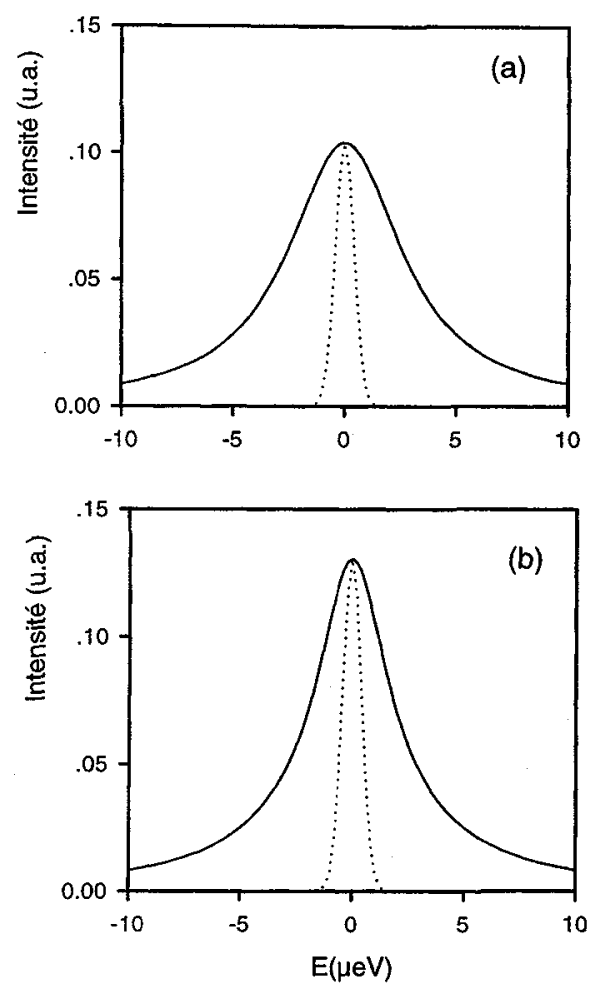

Figure 16. Spectres simulés pour une diffusion (a) isotrope, et (b) anisotrope. Voir la figure précédente pour les commentaires.

ainsi que celle de l'isobutane, l'alcane branché le plus simple, a été récemment mesurée sur IN16 [58,62]. Il est intéressant de noter, pour les applications éventuelles des membranes silicalite, que la diffusion des alcanes linéaires est beaucoup plus rapide que celle des branchés, le coefficient de diffusion du n-butane étant de 3 ordres de grandeur supérieur à celui de l'isobutane [62].

\subsection{Systèmes unidimensionnels}

La diffusion du méthane dans la mordénite, une zéolithe à canaux 1D, mais avec des poches latérales, a été étudiée par DQEN dans le but de mesurer une diffusion 1D le long des canaux [63]. En fait l'interprétation des spectres a montré qu'une large proportion des molécules étaient piégées dans les poches latérales, en accord avec des simulations de type Monte Carlo [64,65].

Les premières mesures de diffusion $1 \mathrm{D}$ réalisées par DQEN ont été obtenues pour le méthane dans l' $^{\prime} \mathrm{AlPO}_{4}-5$ [21,66]. A priori, il n'était pas facile de deviner si la diffusion allait être simplement $1 \mathrm{D}$ ou à la file indienne car le diamètre libre que l'on rapporte dans la littérature pour l'AlPO ${ }_{4}-5$ est de $7,3 \AA$, il est basé sur un rayon de 1,35 ̊̊ pour l'atome d'oxygène [67]. Comme le diamètre cinétique du méthane est de 3,8 $\AA$, les molécules pourraient avoir du mal à se croiser. En fait, deux séries de mesures effectuées par PFG NMR sont contradictoires : un groupe en a déduit que la diffusion du méthane était unidirectionnelle mais normale [68] alors que l'autre groupe a conclu à une diffusion à la file indienne [20]. Il est possible que ces désaccords soient liés à la provenance des échantillons. Les isothermes d'adsorption ont montré 

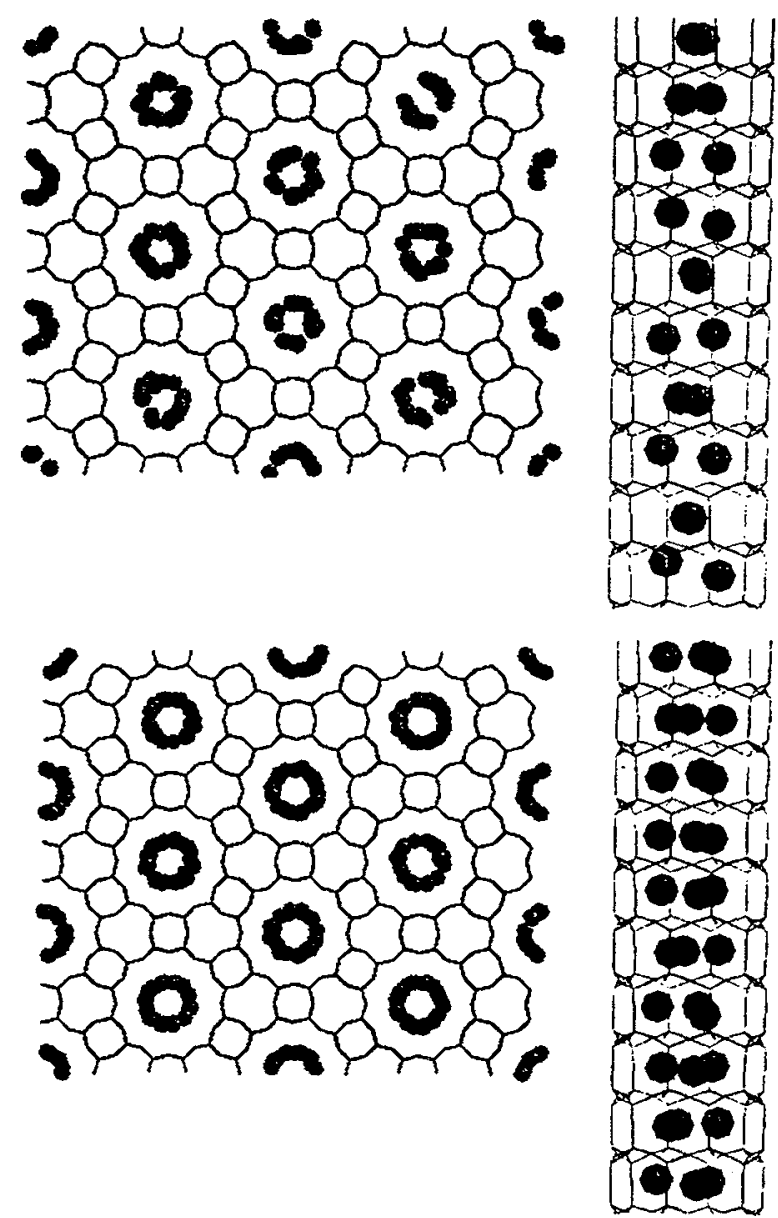

Figure 17. Deux phases différentes obtenues par simulation pour du méthane dans l'AlPO $4^{-5}$ [71]. Les sphères reprễsentent les molécules de méthane, les lignes délimitent le volume des canaux. Les figures du haut correspondent à $4 \mathrm{CH}_{4} / \mathrm{maille}$, celles du bas à $6 \mathrm{CH}_{4} /$ maille. Les canaux sont vus de dessus dans les figures de gauche et de côté dans les figures de droite.

que certains échantillons adsorbaient jusqu'à 4 molécules de méthane par maille et d'autres jusqu'à 6 [69]. Deux simulations ont été réalisées sur ce système pour étudier l'adsorption du méthane dans ce matériau, en utilisant différents potentiels [70,71]. Les simulations permettent d'expliquer comment sont disposées les molécules à saturation (Figure 17), on voit que l'on arrive à placer jusqu'à 3 molécules de méthane dans un même plan. Le diamètre libre de $1^{\prime} \mathrm{AlPO}_{4}-5$ est ainsi bien plus grand que la valeur nominale, il a été estimé à $8,2 \AA[72]$.

Les spectres DQEN obtenus pour plusieurs concentrations de méthane dans cette structure, et à différentes températures, ont été interprétés par le modèle de diffusion 1D normale $[21,66]$. Une comparaison entre spectres expérimentaux et calculés est montrée sur la Figure 18. Tous les spectres ont été affinés simultanément en prenant la moyenne de poudre de la fonction de diffusion ID normale, équation 34, convoluée avec la résolution expérimentale. On a tenu compte également d'une rotation isotrope des molécules, convoluée avec la résolution de l'instrument. En comparant les facteurs de confiance obtenus pour divers modèles, il apparaît que l'accord avec le modèle de diffusion ID est meilleur de quelques \% par rapport à une diffusion 3D, le modèle $1 \mathrm{D}$ à la file indienne ne fittant pas du 


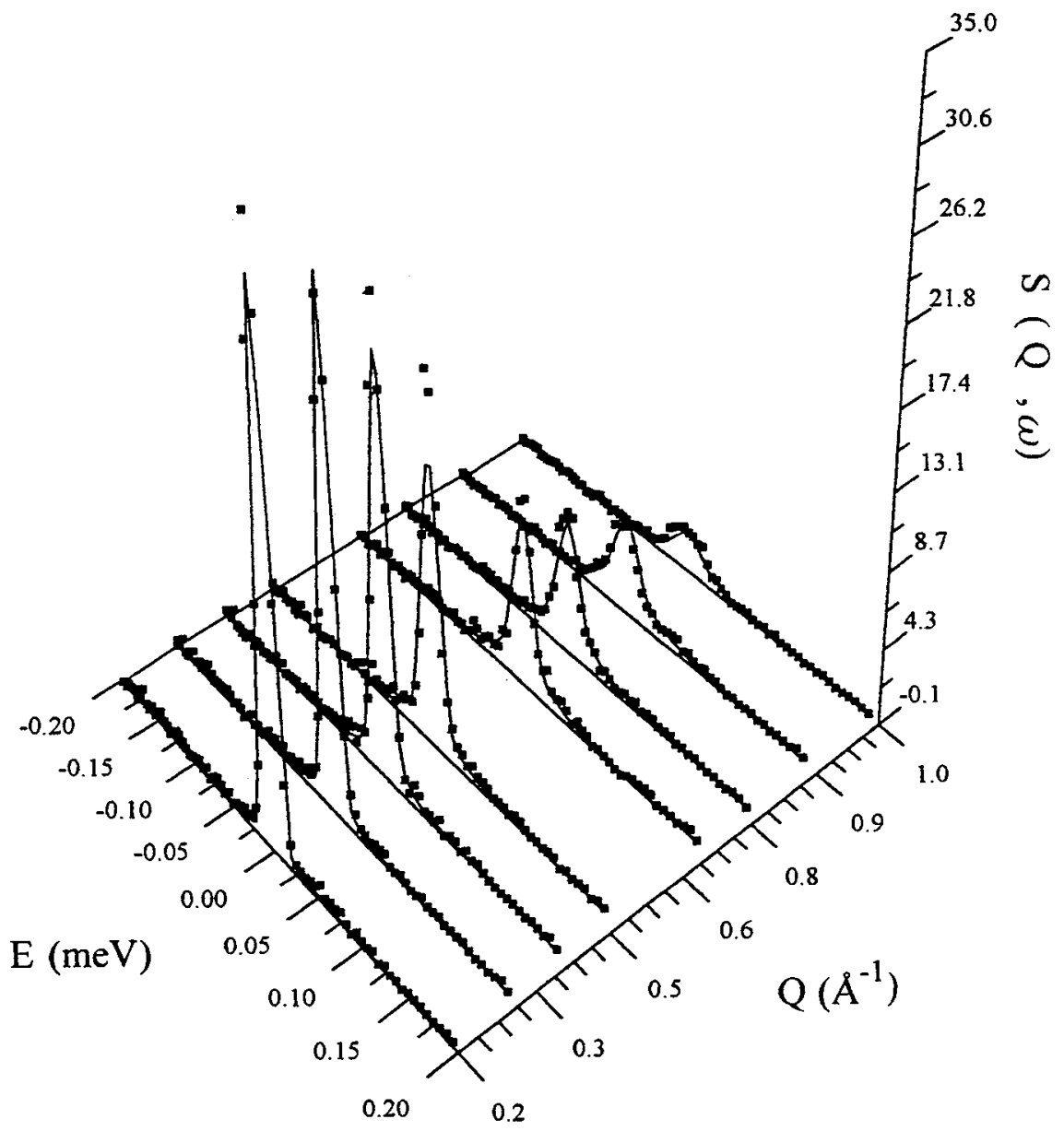

Figure 18. Spectres DQEN, obtenus à différentes valeurs du transfert de moment $Q$, pour du méthane dans 1 'AIPO $4-5(1$ molécule par maille, $T=155 \mathrm{~K}$ ) : (目) points expérimentaux, la ligne continue a été obtenue pour un modèle de diffusion 1D normale.

tout. Les facteurs de confiance sont bien moins bons sur $\mathbb{I N 5}=30 \%$, que sur IN6, cf. section précédente, car le flux est plus faible. Le coefficient de diffusion obtenu par DQEN à $155 \mathrm{~K}$, pour une concentration de 0,7 molécules par maille, $1,6 \times 10^{-9} \mathrm{~m}^{2} \mathrm{~s}^{-1}$ [21], est en bon accord avec celui déterminé à $300 \mathrm{~K}$ par PFG NMR, pour la même concentration : $2,9 \times 10^{-9} \mathrm{~m}^{2} \mathrm{~s}^{-1}$ [68]. Pour de plus fortes concentrations en méthane, les coefficients de diffusion déduits des spectres DQEN sont comparables : $1,0 \times 10^{-9} \mathrm{~m}^{2} \mathrm{~s}^{-1}$ à $97 \mathrm{~K}$ pour 1,2 molécules par maille [66], et $1,2 \times 10^{-9} \mathrm{~m}^{2} \mathrm{~s}^{-1}$ à $155 \mathrm{~K}$ pour 1 molécule par maille [21]. Des simulations de dynamique moléculaire réalisées récemment pour ce système penchent également pour une diffusion 1D normale [73]. Cependant, il a été trouvé dans cette étude théorique que les molécules d'éthane se croisaient difficilement et présentaient une mobilité intermédiaire entre une diffusion 1D normale et une diffusion à la file indienne. Le même groupe a déduit de mesures PFG NMR que l'éthane diffusait à la file indienne [74] alors que nous avons trouvé par DQEN que cette molécule diffusait normalement en 1D, 

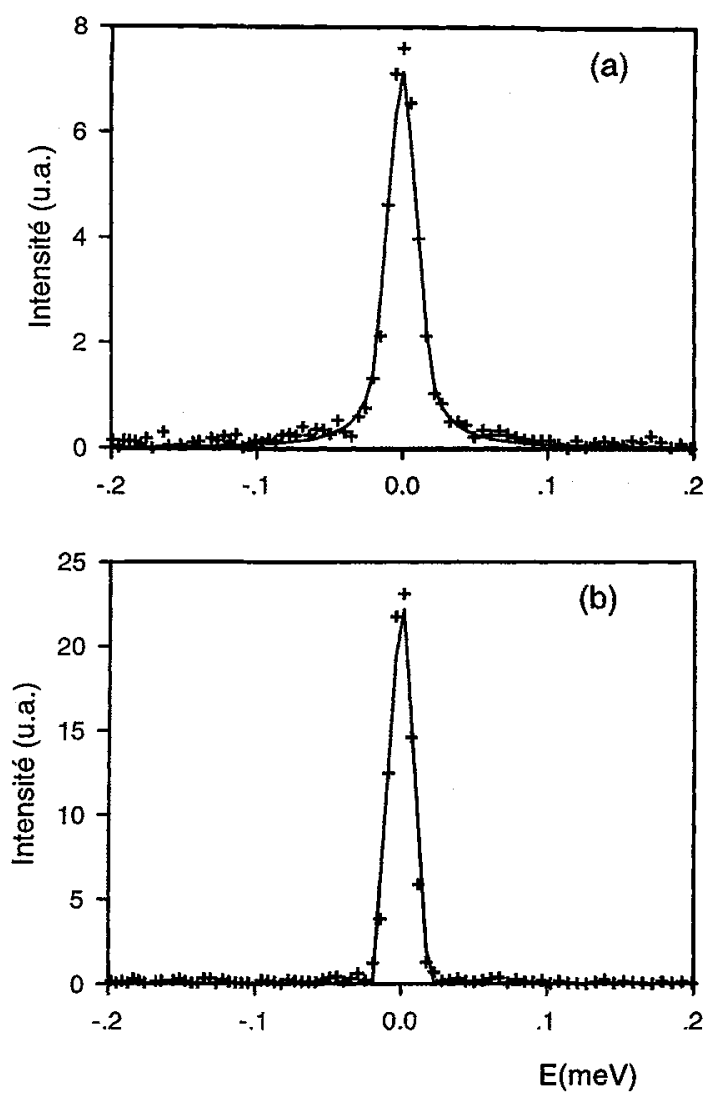

Figure 19. Comparaison de spectres expérimentaux $(+)$ et calculés $(-$ obtenus pour du cyclopropane dans 1 'AlPO $4-5(\mathrm{~T}=273$ $\mathrm{K}, \mathrm{Q}=0,39 \AA^{-1}$ ) (a) 0,6 molécule par maille, la ligne continue a été calculée avec le modèle de diffusion $1 \mathrm{D}$ normale ; (b) 1,3 molécule par maille, la ligne continue a été calculée avec le modèle de diffusion 1D à la file indienne.

comme le méthane [21]. Il a été mentionné dans le paragraphe précédent que l'origine et le traitement thermique des échantillons pouvaient expliquer ces différences. En effet, la cristallinité de cet aluminophosphate est moins stable que pour une zéolithe, elle se détériore avec le temps en présence d'eau. L'étape de calcination est cruciale car suivant la montée en température, on peut produire ou non des défauts et des effondrements partiels de la structure.

Pour une molécule un peu plus encombrante, comme le cyclopropane, un comportement différent a été trouvé par DQEN [21]. A l'encontre du méthane ou de l'éthane, un effet plus important de la concentration a été constaté. Pour 0,6 molécule par maille, un élargissement notable est obtenu pour une faible valeur de Q, Figure 19(a). Les spectres ont été affinés avec la fonction de diffusion 1D normale, le coefficient de diffusion étant de $1,8 \times 10^{-9} \mathrm{~m}^{2} \mathrm{~s}^{-1}$ à $273 \mathrm{~K}$. Par contre pour 1,3 molécule par maille, le pic est strictement élastique, Figure 19(b). Un affinement de ce spectre avec le modèle de diffusion 1D à la file indienne indique que le facteur de mobilité est plus petit que $10^{-12} \mathrm{~m}^{2} \mathrm{~s}^{-1 / 2}$. Cet effet important de la concentration indique que la diffusion du cyclopropane dans l' $^{\prime} \mathrm{AlPO}_{4}-5$ est probablement à la file indienne mais le facteur de mobilité est trop faible pour pouvoir être mésuré avec cette résolution. 

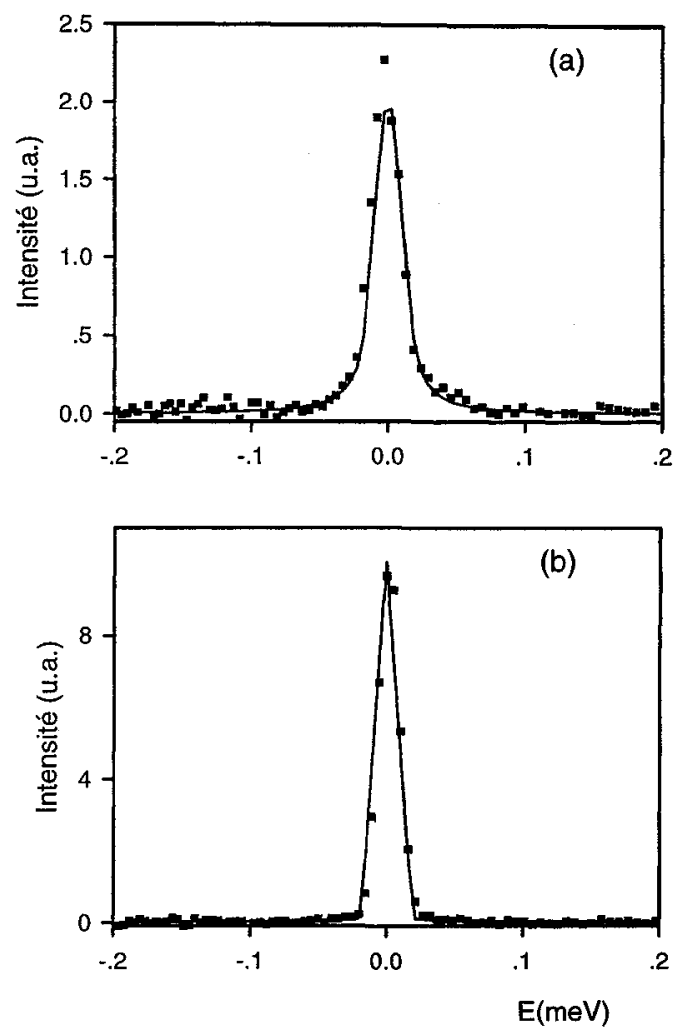

Figure 20. Comparaison de spectres expérimentaux (a) et calculés $(-$ ) obtenus pour du méthane dans la ZSM-48 (T $=155 \mathrm{~K}$, $Q=0,35 \AA^{-1}$ ) (a) 0,11 molécule par maille, la ligne continue a été calculée avec le modèle de diffusion 1D normale ; (b) 0,48 molécule par maille, la ligne continue a été calculée avec le modèle de diffusion $1 \mathrm{D}$ à la file indienne.

Ce mouvement de diffusion 1D à la file indienne a été mis en évidence de façon plus claire en étudiant la diffusion du méthane dans la ZSM-48 [21]. Cette zéolithe a des canaux 1D de plus petite

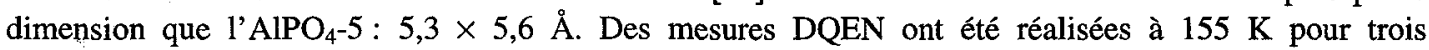
concentrations en méthane. Pour le taux de remplissage le plus faible : $\theta=0,11$, un élargissement important est mesuré à petit $\mathrm{Q}$, Figure 20(a). Pour cette valeur de $\mathrm{Q}\left(0,35 \AA^{-1}\right)$ seulement $5 \%$ de l'intensité vient de la rotation de telle sorte que l'on est sûr que l'élargissement est dû à la translation. Le meilleur affinement a été obtenu avec le modèle de diffusion 1D normale, le coefficient de diffusion étant de $2,5 \times 10^{-9} \mathrm{~m}^{2} \mathrm{~s}^{-1}$. Pour un taux de remplissage intermédiaire, $\theta=0,48$, le spectre obtenu pour une même valeur de $\mathrm{Q}$ est complètement différent, Figure 20(b). Cet effet important de la concentration sur la mobilité du méthane, qui n'avait pas été observé dans l' $\mathrm{AlPO}_{4}-5$, est une indication de diffusion à la file indienne. Le pied quasi-élastique dans la Figure 20 (b) peut seulement être fitté par ce modèle, équation 44 , le facteur de mobilité étant de $2 \times 10^{-12} \mathrm{~m}^{2} \mathrm{~s}^{-1 / 2}$. Pour un taux de remplissage plus élevé, $\theta=0,8$, le spectre est élastique, comme dans la Figure 19(b). Pour la concentration la plus élevée en méthane, le facteur de mobilité est par conséquent plus petit que $10^{-12} \mathrm{~m}^{2} \mathrm{~s}^{-1 / 2}$.

Pour le méthane dans la ZSM-48, on observe ainsi deux régimes différents de diffusion : une diffusion 1D normale pour un faible taux de remplissage, et une diffusion 1D à la file indienne pour un taux de remplissage moyen. La transition entre les deux régimes est reliée à la variation du rapport entre la distance moyenne entre 2 molécules, $\ell$, et l'échelle de distance $\lambda$ correspondant à l'expérience $(\lambda=2 \pi / Q$ 
$=18 \AA$ pour les spectres de la Figure 20). A faible concentration, $\theta=0,11, \ell$ est supérieur à $\lambda$ de telle sorte qu'à l'échelle de temps de l'expérience, les molécules diffusent de façon indépendante, sans interaction avec les autres molécules. Par contre pour un taux de remplissage moyen, $\theta=0,48, \ell$ est inférieur à $\lambda$. Dans ce cas, il se produit des collisions entre les molécules et les conditions pour observer une diffusion à la file indienne sont remplies.

\section{CONCLUSION}

Les phénomènes de diffusion à l'échelle microscopique suscitent encore un intérêt certain, et l'utilisation de nouveaux matériaux comme les membranes zéolithes nécessitent une connaissance détaillée des phénomènes de transport. Parmi les nombreuses techniques utilisées, la DQEN reste une méthode bien adaptée. Le faible nombre de spectromètres neutron disponibles dans le monde limite le nombre de mesures, par rapport à des techniques comme la RMN. Malgré tout, les échelles de temps et de distance couvertes par la DQEN sont telles que les résultats obtenus par cette technique sont souvent uniques et constituent une base de données indispensable pour tester les méthodes de simulation.

\section{Références}

1. Fick A. E., Ann. Phys. 94 (1855) 59.

2. Cranck J., Mathematics of Diffusion (Oxford Press, London, 1975).

3. Cook J. C., Richter D., Schärpf O., Benham M. J., Ross D. K., Hempelmann R., Anderson I. S., Sinha S. K., J. Phys. : Condens. Matter, 2 (1990) 79.

4. Jobic H., Kärger J., Bée M., Phys. Rev. Lett. 82 (1999) 4260.

5. Bée M. (chapitre d'introduction).

6. Einstein A., Ann. Phys. 17 (1905) 349.

7. Hall P. L., Ross D. K., Mol. Phys. 42 (1981) 673.

8. Chudley C. T., Elliott R. J., Proc. Phys. Soc. London 77 (1961) 353.

9. Rowe J. M., Sköld K., Flotow H. E., Rush J. J., J. Phys. Chem. Solids 32 (1971) 41.

10. Conroy H., J. Chem. Phys. 47 (1967) 5307.

11. Suzukawa Jr., H. H., Wolfsberg M., J. Chem. Phys. 59 (1973) 3992.

12. Singwi K. S., Sjölander A., Phys. Rev. 119 (1960) 863.

13. Egelstaff P. A., An Introduction to the Liquid State (Academic, London, 1967).

14. June R. L., Bell A. T., Theodorou D. N., J. Phys. Chem. 94 (1990) 1508.

15. Hall P. L., Ross D. K., Mol. Phys. 36 (1978) 1549.

16. Volino F., Dianoux A. J., Mol. Phys. 41 (1980) 271.

17. Dianoux A. J., Pinéri M., Volino F., Mol. Phys. 46 (1982) 129.

18. Dianoux A. J., Volino F., Hervet H., Mol. Phys. 30 (1975) 1181.

19. Hodkin A. L., Keynes R. D., J. Physiol. (London) 128 (1955) 61.

20. Hahn K., Kärger J., Kukla V., Phys. Rev. Letters 76 (1996) 2762.

21. Jobic H., Hahn K., Kärger J., Bée M., Tuel A., Noack M., Girnus I., Kearley G. J., J. Phys. Chem. B 101 (1997) 5834.

22. Jobic H., Bée M., Renouprez A., Surf. Sci. 140 (1984) 307.

23. Hahn K., Jobic H., Kärger J., Phys. Rev. E 59 (1999) 6662. 
24. Hahn K., Kärger J., J. Phys. A 28 (1995) 3061.

25. Abramovitz M., Stegun I. A., Handbook of Mathematical Functions (Dover Publications, NewYork, 1968).

26. Fitch A. N., Jobic H., Molecular Sieves, H. G. Karge et J. Weitkamp, Eds. (Springer, Heidelberg, 1999) Vol. II, pp.31.

27. D. W. Breck, Zeolite Molecular Sieves (Wiley, New York, 1974).

28. Kärger J., Ruthven D. M., Diffusion in Zeolites and Other Microporous Solids (Wiley, New York, 1992).

29. Casanave D., Giroir-Fendler A., Sanchez J., Loutaty R., Dalmon J. A., Catal. Today 25 (1995) 309.

30. Kahn R., Cohen de Lara E., Viennet E., J. Chem. Phys. 91 (1989) 5097.

31. Stockmeyer R., Zeolites 5 (1985) 393.

32. Stockmeyer R., Zeolites 12 (1992) 251.

33. Kärger J. et al. (en préparation).

34. Martin C., Coulomb J. P., Ferrand M., Europhys. Lett. 36 (1996) 503.

35. Jobic H., Bée M., Kearley G. J., Zeolites 9 (1989) 312.

36. Jobic H., Bée M., Kearley G. J., Zeolites 12 (1992) 146.

37. Jobic H., Bée M., Caro J., Proceedings $9^{\text {th }}$ Int. Zeol. Conf., Montréal, 1992 ; R von Ballmoos, J. B.

Higgins et M. M. J. Treacy, Eds. (Butterworth-Heinemann, Boston, 1993) Vol. II, pp. 121.

38. Caro J., Bülow M., Schirmer W., Kärger J., Heink W., Pfeifer J., Zdanov S. P., J. Chem. Soc. Faraday Trans. 1, 81 (1985) 2541.

39. Heink W., Kärger J., Pfeifer H., Datema K. P., Nowak A. K., J. Chem. Soc. Faraday Trans. 88 (1992) 3505.

40. Demontis P., Fois E. S., Suffriti G. B., Quartieri S., J Phys. Chem. 94 (1990) 4329.

41. June R. L., Bell A. T., Theodorou D. N., J. Phys. Chem. 94 (1990) 8232.

42. Demontis P., Suffriti G. B., Fois E. S., Quartieri S., J Phys. Chem. 96 (1992) 1482.

43. June R. L., Bell A. T., Theodorou D. N., J. Phys. Chem. 96 (1992) 1051.

44. Nowak A. K., den Ouden C. J. J., Pickett S. D., Smit B., Cheetham A. K., Post M. F. M., Thomas J. M., J. Phys. Chem. 95 (1991) 848.

45. Catlow C. R. A., Freeman C. M., Vessal B., Tomlinson S. M., Leslie M., J. Chem. Soc. Faraday Trans. 87 (1991) 1947.

46. Goodbody S. J., Watanabe K., MacGowan D., Walton J. P. R. B., Quirke N., J. Chem. Soc. Faraday Trans. 87 (1991) 1951.

47. Hernandez E., Catlow C. R. A., Proc. R. Soc. Lond. A 448 (1195) 143.

48. Maginn E. J., Bell A. T., Theodorou D. N., J. Phys. Chem. 100 (1996) 7155.

49. George A. R., Catlow C. R. A., Thomas J. M., Microporous Materials 11 (1997) 97.

50. Van-Den-Begin N., Rees L. V. C., Caro J., Bülow M., Zeolites 9 (1989) 287.

51. Shen D., Rees L. V. C., Caro J., Bülow M., Zibrowius B., Jobic H., J. Chem. Soc. Faraday Trans. 86 (1990) 3943.

52. Hayhurst D. T., Paravar A. R., Zeolites 8 (1988) 27.

53. Talu O., Sun M. S., Shah D. B., AIChE Journal 44 (1998) 681.

54. Millot B. et al. (en préparation).

55. Hufton J. R., Danner R. P., AIChE Journal 39 (1993) 962.

56. Jama M. A., Delmas M. P. F., Ruthven D. M., Zeolites 18 (1997) 200.

57. Eic M., Ruthven D. M., Studies in Surf. Sci. Catal. 49 B (1989) 897.

58. Stepanov A. G., Shubin A. A., Luzgin M. V., Jobic H., Tuel A., J. Phys. Chem. B 102 (1998) 10860.

59. Stockmeyer R., Ber. Bunsenges. Physik Chem. 80 (1976) 625.

60. Trouw F., Spectrochim. Acta 48A (1992) 455.

61. Zibrowius B., Caro J., Kärger J., Z. phys. Chemie Leipzig 269 (1988) 1101.

62. Millot B., Méthivier A., Jobic H., Moueddeb H., Bée M., J. Phys. Chem. B 103 (1999) 1096.

63. Jobic H., Bée M., Z. Phys. Chem. 189 (1995) 179.

64. Smit B., den Ouden C. J. J., J. Phys. Chem. 92 (1988) 7169. 
65. George A. R., Catlow C. R. A., Thomas J. M., Microporous Materials 11 (1997) 97.

66. Martin C., Coulomb J. P., Grillet Y., Kahn R., 'Fundamentals of Adsorption', M. D. Le Van, Ed., (Kluwer Academic Publishers, Boston, 1996) p. 587.

67. Meier W. M., Olson D. H., Baerlocher Ch., Eds., Atlas of Zeolites Structure Types, Elsevier, Amsterdam, Zeolites, 17, 1996.

68. Nivarthi S. S., Mc. Cormik A. V., Davies H. T., Chem. Phys. Lett. 229 (1994) 297.

69. Coulomb J. P., Martin C., Grillet Y., Tosi-Pellenq N., Studies in Surface Science and Catalysis, (Elsevier, Amsterdam, 1994) Vol.84, p.445.

70. Lachet V., Boutin A., Pellenq R. J., Nicholson D., Fuchs A., J. Phys. Chem. 100 (1996) 9006.

71. Maris T., Vlugt T. J. H., Smit B., J. Phys. Chem. B 102 (1998) 7183.

72. Martin C., Tosi-Pellenq N., Patarin J., Coulomb J. P., Langmuir (sous presse).

73. Keffer D., Mc. Cormik A. V., Davies H. T., Mol. Phys. 87 (1996) 367.

74. Gupta V., Nivarthi S. S., Mc. Cormik A. V., Davies H. T., Chem. Phys. Lett. 247 (1995) 596. 